\title{
Refined analytic torsion on manifolds with boundary
}

\author{
BORIS VERTMAN
}

\begin{abstract}
We discuss the refined analytic torsion, introduced by $\mathrm{M}$ Braverman and T Kappeler as a canonical refinement of analytic torsion on closed manifolds. Unfortunately there seems to be no canonical way to extend their construction to compact manifolds with boundary. We propose a different refinement of analytic torsion, similar to Braverman and Kappeler, which does apply to compact manifolds with and without boundary. In a subsequent publication we prove a surgery formula for our construction.
\end{abstract}

$58 \mathrm{~J} 52$

\section{Introduction}

The refined analytic torsion, defined by M Braverman and T Kappeler [8; 6] on closed manifolds, can be viewed as a refinement of the Ray-Singer torsion, since it is a canonical choice of an element with Ray-Singer norm one, in case of unitary representations.

The complex phase of the refinement is given by the rho-invariant of the odd-signature operator. Hence one can expect the refined analytic torsion to give more geometric information than the Ray-Singer torsion.

This is indeed the case in the setup of lens spaces with explicit formulas for the associated Ray-Singer torsion and eta-invariants; see Huang [20, Section 5] and the references therein. There it is easy to find explicit examples of lens spaces which are not distinguished by the Ray-Singer torsion, however have different rho-invariants of the associated odd-signature operators.

An important property of the Ray-Singer torsion norm is its gluing property, as established by W Lück [23] and S Vishik [33]. It is natural to expect a refinement of the Ray-Singer torsion to admit an analogous gluing property.

Unfortunately there seems to be no canonical way to extend the construction of Braverman and Kappeler to compact manifolds with boundary. In particular a gluing formula seems to be out of reach.

We propose a different refinement of analytic torsion, similar to Braverman and Kappeler, which does apply to compact manifolds with and without boundary. In the subsequent 
publication [32] we establish a gluing formula for our construction, which in fact can also be viewed as a gluing law for the original definition of refined analytic torsion by Braverman and Kappeler.

The presented construction is analogous to the definition in [8; 6], but refines the square of Ray-Singer torsion, in contrast to the construction by Braverman and Kappeler, which refines the Ray-Singer torsion itself. The doubling is essential to allow the extension of the concepts to any smooth compact Riemannian manifold, with or without boundary.

In case of empty boundary our construction can be identified with the square of refined analytic torsion by Braverman and Kappeler, up to a defect in terms of a difference of certain eta-invariants. Nevertheless we still refer to our concept as "refined analytic torsion" within the present discussion.

Acknowledgements The results of this article were obtained during the author's $\mathrm{PhD}$ studies at Bonn University, Germany. The author would like to thank his thesis advisor Professor Matthias Lesch for his support and useful discussions. The author would also like to express his gratitude to the anonymous referee for valuable comments which greatly helped to improve the presentation of the article. The author was supported by the German Research Foundation as a scholar of the Graduiertenkolleg 1269 "Global Structures in Geometry and Analysis".

\section{Motivation for the generalized construction}

The essential ingredient in the definition of the refined analytic torsion in [6] is the twisted de Rham complex with a chirality operator and the elliptic odd-signature operator associated to the complex, viewed as a map between the even forms. Hence in the case of a manifold with boundary we are left with the task of finding elliptic boundary conditions for the odd-signature operator which preserve the complex structure and provide a Fredholm complex, in the sense of [9].

The notions of a Hilbert and a Fredholm complex were studied systematically in [9] and will be provided for convenience in the forthcoming section. The boundary conditions, that give rise to a Hilbert complex are referred to as "ideal boundary conditions". It is important to note that the most common self-adjoint extensions of the odd-signature operator between the even forms do not come from ideal boundary conditions.

The existence and explicit determination of elliptic boundary conditions for the oddsignature operator between the even forms, arising from ideal boundary conditions, is 
an open question. However, it is clear that the absolute and relative boundary conditions do not satisfy these requirements.

On the other hand the gluing formula in [33] and [23] for the Ray-Singer torsion makes essential use of the relative and absolute boundary conditions. Since the establishment of a corresponding gluing formula for the refined analytic torsion is a motivation for our discussion, these boundary conditions seem to be natural choices.

We are left with a dilemma, since neither the relative nor the absolute boundary conditions are invariant under the Hodge operator. We resolve this dilemma by combining the relative and absolute boundary conditions. This allows us to apply the concepts of [6] in a new setting and to establish the desired gluing formula.

\section{Definition of refined analytic torsion}

Let $\left(M^{m}, g^{M}\right)$ be a smooth compact connected odd-dimensional oriented Riemannian manifold with boundary $\partial M$, which may be empty. Let $\left(E, \nabla, h^{E}\right)$ be a flat complex vector bundle with any fixed Hermitian metric $h^{E}$, which need not to be flat with respect to $\nabla$.

The flat covariant derivative $\nabla$ is a first order differential operator

$$
\nabla: \Gamma(E) \rightarrow \Gamma\left(T^{*} M \otimes E\right),
$$

satisfying the Leibniz rule

$$
\nabla_{X}(f s)=(X f) s+f \nabla_{X} s, \quad s \in \Gamma(E), X \in \Gamma(T M), f \in C^{\infty}(M) .
$$

The covariant derivative $\nabla$ extends by the Leibniz rule to the twisted exterior differential $\nabla: \Omega_{0}^{k}(M, E) \rightarrow \Omega_{0}^{k+1}(M, E)$ on $E$-valued differential forms with compact support in the interior of the manifold $\Omega_{0}^{k}(M, E)$. The exterior differential satisfies the (generalized) Leibniz rule

$$
\nabla_{X}(w \wedge \eta)=\left(\nabla_{X} w\right) \wedge \eta+(-1)^{p} w \wedge \nabla_{X} \eta,
$$

for any $w \in \Omega_{0}^{p}(M), \eta \in \Omega_{0}^{q}(M, E), X \in \Gamma(T M)$.

Due to flatness of $(E, \nabla)$ the twisted exterior differential gives rise to the twisted de Rham complex $\left(\Omega_{0}^{*}(M, E), \nabla\right)$. The metrics $g^{M}, h^{E}$ induce an $L^{2}-$ inner product on $\Omega_{0}^{*}(M, E)$. We denote the $L^{2}$ - completion of $\Omega_{0}^{*}(M, E)$ by $L_{*}^{2}(M, E)$.

Next we introduce the notion of the dual covariant derivative $\nabla^{\prime}$. It is defined by requiring

$$
d h^{E}(u, v)[X]=h^{E}\left(\nabla_{X} u, v\right)+h^{E}\left(u, \nabla_{X}^{\prime} v\right)
$$


to hold for all $u, v \in C^{\infty}(M, E)$ and $X \in \Gamma(T M)$. In the special case that the Hermitian metric $h^{E}$ is flat with respect to $\nabla$, the dual $\nabla^{\prime}$ and the original covariant derivative $\nabla$ coincide. More precisely the Hermitian metric $h^{E}$ can be viewed as a section of $E^{*} \otimes E^{*}$. The covariant derivative $\nabla$ on $E$ gives rise to a covariant derivative on the tensor bundle $E^{*} \otimes E^{*}$, also denoted by $\nabla$ by a minor abuse of notation.

For $u, v, X$ as above one has

$$
\nabla h^{E}(u, v)[X]=d h^{E}(u, v)[X]-h^{E}\left(\nabla_{X} u, v\right)-h^{E}\left(u, \nabla_{X} v\right) .
$$

In view of (3-1) we find

$$
\nabla h^{E}=0 \Leftrightarrow \nabla=\nabla^{\prime} .
$$

As before, the dual $\nabla^{\prime}$ gives rise to a twisted de Rham complex. Consider the differential operators $\nabla, \nabla^{\prime}$ and their formal adjoint differential operators $\nabla^{t}, \nabla^{\prime t}$. The associated minimal closed extensions $\nabla_{\min }, \nabla_{\min }^{\prime}$ and $\nabla_{\min }^{t}, \nabla_{\min }^{\prime t}$ are defined as the graph-closures in $L_{*}^{2}(M, E)$ of the respective differential operators. The maximal closed extensions are defined by

$$
\nabla_{\max }:=\left(\nabla_{\min }^{t}\right)^{*}, \quad \nabla_{\max }^{\prime}:=\left(\nabla_{\min }^{\prime t}\right)^{*} .
$$

These extensions define Hilbert complexes in the following sense, as introduced in [9].

Definition 3.1 [9] Let the Hilbert spaces $H_{i}, i=0, \ldots, m, H_{m+1}=\{0\}$ be mutually orthogonal. For each $i=0, \ldots, m$ let $D_{i} \in C\left(H_{i}, H_{i+1}\right)$ be a closed operator with domain $\mathcal{D}\left(D_{i}\right)$ dense in $H_{i}$ and range in $H_{i+1}$. Put $\mathcal{D}_{i}:=\mathcal{D}\left(D_{i}\right)$ and $R_{i}:=D_{i}\left(\mathcal{D}_{i}\right)$ and assume

$$
R_{i} \subseteq \mathcal{D}_{i+1}, \quad D_{i+1} \circ D_{i}=0 .
$$

This defines a complex $(\mathcal{D}, D)$

$$
0 \rightarrow \mathcal{D}_{0} \stackrel{D_{0}}{\longrightarrow} \mathcal{D}_{1} \stackrel{D_{1}}{\longrightarrow} \cdots \stackrel{D_{m-1}}{\longrightarrow} \mathcal{D}_{m} \rightarrow 0 .
$$

Such a complex is called a Hilbert complex. If the homology of the complex is finite, ie if $R_{i}$ is closed and $\operatorname{ker} D_{i} / \operatorname{im} D_{i-1}$ is finite-dimensional for all $i=0, \ldots, m$, the complex is referred to as a Fredholm complex.

Indeed, by [9, Lemma 3.1] the extensions define Hilbert complexes as follows:

$$
\begin{array}{ll}
\left(\mathcal{D}_{\min }, \nabla_{\min }\right), & \text { where } \mathcal{D}_{\text {min }}:=\mathcal{D}\left(\nabla_{\min }\right), \\
\left(\mathcal{D}_{\text {max }}, \nabla_{\max }\right), & \text { where } \mathcal{D}_{\text {max }}:=\mathcal{D}\left(\nabla_{\max }\right) ; \\
\left(\mathcal{D}_{\text {min }}^{\prime}, \nabla_{\text {min }}^{\prime}\right), & \text { where } \mathcal{D}_{\text {min }}^{\prime}:=\mathcal{D}\left(\nabla_{\text {min }}^{\prime}\right), \\
\left(\mathcal{D}_{\text {max }}^{\prime}, \nabla_{\text {max }}^{\prime}\right), & \text { where } \mathcal{D}_{\text {max }}^{\prime}:=\mathcal{D}\left(\nabla_{\text {max }}^{\prime}\right) .
\end{array}
$$


The Laplace operators, associated to Hilbert complexes $\left(\mathcal{D}_{\min }, \nabla_{\min }\right)$ and $\left(\mathcal{D}_{\max }, \nabla_{\max }\right)$, are respectively defined as follows:

$$
\begin{aligned}
\triangle_{\text {rel }}: & =\nabla_{\min }^{*} \nabla_{\min }+\nabla_{\min } \nabla_{\min }^{*}, \\
\mathcal{D}\left(\triangle_{\text {rel }}\right) & =\left\{\omega \in \mathcal{D}\left(\nabla_{\min }\right) \cap \mathcal{D}\left(\nabla_{\min }^{*}\right) \mid \nabla_{\min } \omega \in \mathcal{D}\left(\nabla_{\min }^{*}\right), \nabla_{\min }^{*} \omega \in \mathcal{D}\left(\nabla_{\min }\right)\right\} . \\
\triangle_{\mathrm{abs}} & :=\nabla_{\max }^{*} \nabla_{\max }+\nabla_{\max } \nabla_{\max }^{*}, \\
\mathcal{D}\left(\triangle_{\mathrm{abs}}\right) & =\left\{\omega \in \mathcal{D}\left(\nabla_{\max }\right) \cap \mathcal{D}\left(\nabla_{\max }^{*}\right) \mid \nabla_{\max } \omega \in \mathcal{D}\left(\nabla_{\max }^{*}\right), \nabla_{\max }^{*} \omega \in \mathcal{D}\left(\nabla_{\max }\right)\right\} .
\end{aligned}
$$

The Laplace operators $\triangle_{\text {rel }}^{\prime}$ and $\triangle_{\text {abs }}^{\prime}$ of the Hilbert complexes $\left(\mathcal{D}_{\min }^{\prime}, \nabla_{\min }^{\prime}\right)$ and $\left(\mathcal{D}_{\max }^{\prime}, \nabla_{\max }^{\prime}\right)$ respectively, are defined in a similar way. Note the following well-known central result on these complexes and their Laplacians.

Theorem 3.2 The Hilbert complexes $\left(\mathcal{D}_{\min }, \nabla_{\min }\right)$ and $\left(\mathcal{D}_{\max }, \nabla_{\max }\right)$ are Fredholm with the associated Laplacians $\triangle_{\text {rel }}$ and $\triangle_{\text {abs }}$ being strongly elliptic in the sense of [17]. The de Rham isomorphism identifies the homology of the complexes with the relative and absolute cohomology with coefficients:

$$
\begin{aligned}
& H^{*}\left(\mathcal{D}_{\min }, \nabla_{\min }\right) \cong H^{*}(M, \partial M, E), \\
& H^{*}\left(\mathcal{D}_{\max }, \nabla_{\max }\right) \cong H^{*}(M, E) .
\end{aligned}
$$

Moreover the cohomology of the Fredholm complexes $\left(\mathcal{D}_{\min }, \nabla_{\min }\right)$ and $\left(\mathcal{D}_{\max }, \nabla_{\max }\right)$ can be computed from the smooth subcomplexes

$$
\begin{array}{ll}
\left(\Omega_{\min }^{*}(M, E), \nabla\right), & \Omega_{\min }^{*}(M, E):=\left\{\omega \in \Omega^{*}(M, E) \mid \iota^{*}(\omega)=0\right\}, \\
\left(\Omega_{\max }^{*}(M, E), \nabla\right), & \Omega_{\max }^{*}(M, E):=\Omega^{*}(M, E),
\end{array}
$$

respectively, where we denote by $\iota: \partial M \hookrightarrow M$ the natural inclusion of the boundary.

In the untwisted setup this theorem is essentially the statement of [9, Theorem 4.1]. The theorem remains true in the general setup. An analogue of the trace theorem [25, Theorem 1.9], in case of flat vector bundles, allows an explicit computation of the boundary conditions for $\triangle_{\text {rel }}$ and $\triangle_{\text {abs }}$. Then [17, Lemma 1.11.1] implies strong ellipticity of the Laplacians. Note that this result in the reference [17] is proved explicitly, even though other aspects of [17, Section 1.11] are rather expository.

By strong ellipticity the Laplacians $\triangle_{\text {rel }}$ and $\triangle_{\text {abs }}$ are Fredholm and by [9, Theorem $2.4]$ the complexes $\left(\mathcal{D}_{\min }, \nabla_{\min }\right)$ and $\left(\mathcal{D}_{\max }, \nabla_{\max }\right)$ are Fredholm as well. By [9, Theorem 3.5] their cohomology indeed can be computed from the smooth subcomplexes $\left(\Omega_{\min }^{*}(M, E), \nabla\right)$ and $\left(\Omega_{\max }^{*}(M, E), \nabla\right)$, respectively.

Finally, the relation to the relative and absolute cohomology (the twisted de Rham theorem) is proved in [26, Section 4] for flat Hermitian metrics, but an analogous proof 
works in the general case. Corresponding results hold also for the complexes associated to the dual connection $\nabla^{\prime}$.

Furthermore, the Riemannian metric $g^{M}$ and the fixed orientation on $M$ give rise to the Hodge star operator for any $k=0, \ldots, m=\operatorname{dim} M$ :

$$
*: \Omega^{k}(M, E) \rightarrow \Omega^{m-k}(M, E) .
$$

Define

$$
\Gamma:=i^{r}(-1)^{k(k+1) / 2} *: \Omega^{k}(M, E) \rightarrow \Omega^{m-k}(M, E), \quad r:=(\operatorname{dim} M+1) / 2 .
$$

This operator extends to a well-defined self-adjoint involution on $L_{*}^{2}(M, E)$, which we also denote by $\Gamma$. The following properties of $\Gamma$ are essential for the later construction.

Lemma 3.3 The self-adjoint involution $\Gamma$ on $L_{*}^{2}(M, E)$, restricted to the domains of the minimal and maximal closed extension of $\nabla$, acts as follows:

$$
\begin{aligned}
& \left.\Gamma\right|_{\mathcal{D}\left(\nabla_{\min }\right)}: \mathcal{D}\left(\nabla_{\min }\right) \rightarrow \mathcal{D}\left(\left(\nabla_{\max }^{\prime}\right)^{*}\right), \\
& \left.\Gamma\right|_{\mathcal{D}\left(\nabla_{\max }\right)}: \mathcal{D}\left(\nabla_{\max }\right) \rightarrow \mathcal{D}\left(\left(\nabla_{\min }^{\prime}\right)^{*}\right) .
\end{aligned}
$$

With $\Gamma$ restricted to appropriate domains, we have

$$
\left.\left.\Gamma\right|_{\mathcal{D}\left(\nabla_{\min }\right)} \nabla_{\min } \Gamma\right|_{\mathcal{D}\left(\left(\nabla_{\max }^{\prime}\right)^{*}\right)}=\left(\nabla_{\max }^{\prime}\right)^{*},\left.\left.\quad \Gamma\right|_{\mathcal{D}\left(\nabla_{\max }\right)} \nabla_{\max } \Gamma\right|_{\mathcal{D}\left(\left(\nabla_{\min }^{\prime}\right)^{*}\right)}=\left(\nabla_{\min }^{\prime}\right)^{*} .
$$

Proof One first checks explicitly (cf [3, Proposition 3.58])

$$
\Gamma \nabla \Gamma=\left(\nabla^{\prime}\right)^{t}, \quad \Gamma \nabla^{\prime} \Gamma=\nabla^{t} .
$$

Recall that the maximal domain of $\nabla, \nabla^{\prime}$ can also be characterized as a subspace of $L_{*}^{2}(M, E)$ with its image under $\nabla, \nabla^{\prime}$ being again in $L_{*}^{2}(M, E)$. Since $\Gamma$ is an involution on $L_{*}^{2}(M, E)$, we obtain for the action of $\Gamma$ restricted to $\mathcal{D}\left((\nabla)_{\max }^{t}\right)$ and $\mathcal{D}\left(\left(\nabla^{\prime}\right)_{\max }^{t}\right)$

$$
\left.\Gamma\right|_{\mathcal{D}\left(\left(\nabla^{\prime}\right)_{\max }^{t}\right)}: \mathcal{D}\left(\left(\nabla^{\prime}\right)_{\max }^{t}\right) \rightarrow \mathcal{D}\left(\nabla_{\max }\right),\left.\quad \Gamma\right|_{\mathcal{D}\left((\nabla)_{\max }^{t}\right)}: \mathcal{D}\left(\nabla_{\max }^{t}\right) \rightarrow \mathcal{D}\left(\nabla_{\max }^{\prime}\right) .
$$

With $\Gamma$ restricted to appropriate domains, we have

$$
\begin{aligned}
& \left.\left.\Gamma\right|_{\mathcal{D}\left(\nabla_{\max }\right)} \nabla_{\max } \Gamma\right|_{\mathcal{D}\left(\left(\nabla^{\prime}\right)_{\max }^{t}\right)}=\left(\nabla^{\prime}\right)_{\max }^{t} \equiv\left(\nabla_{\text {min }}^{\prime}\right)^{*}, \\
& \left.\left.\Gamma\right|_{\mathcal{D}\left(\nabla_{\max }^{\prime}\right)} \nabla_{\text {max }}^{\prime} \Gamma\right|_{\mathcal{D}\left(\nabla_{\max }^{t}\right)}=\nabla_{\text {max }}^{t} \equiv \nabla_{\text {min }}^{*} .
\end{aligned}
$$

Taking adjoints on both sides of the last relation, we obtain the full statement of the lemma, since $\Gamma$ is self-adjoint. 
Next we introduce the following central concepts. The self-adjoint involution $\Gamma$ gives rise to a self-adjoint involution $\widetilde{\Gamma}$ on $L_{*}^{2}(M, E) \oplus L_{*}^{2}(M, E)$, the "chirality operator", acting by definition antidiagonally with respect to the direct sum of the components

$$
\widetilde{\Gamma}:=\left(\begin{array}{cc}
0 & \Gamma \\
\Gamma & 0
\end{array}\right): L_{*}^{2}(M, E) \oplus L_{*}^{2}(M, E) \rightarrow L_{*}^{2}(M, E) \oplus L_{*}^{2}(M, E) .
$$

\section{Definition 3.4}

$$
\begin{aligned}
(\tilde{\mathcal{D}}, \tilde{\nabla}) & :=\left(\mathcal{D}_{\min }, \nabla_{\min }\right) \oplus\left(\mathcal{D}_{\max }, \nabla_{\max }\right), \text { where } \mathcal{D}_{\min }=\mathcal{D}\left(\nabla_{\min }\right), \mathcal{D}_{\max }=\mathcal{D}\left(\nabla_{\max }\right) \\
\left(\tilde{\mathcal{D}}^{\prime}, \tilde{\nabla}^{\prime}\right) & :=\left(\mathcal{D}_{\text {min }}^{\prime}, \nabla_{\min }^{\prime}\right) \oplus\left(\mathcal{D}_{\max }^{\prime}, \nabla_{\max }^{\prime}\right), \text { where } \mathcal{D}_{\min }^{\prime}=\mathcal{D}\left(\nabla_{\min }^{\prime}\right), \mathcal{D}_{\max }^{\prime}=\mathcal{D}\left(\nabla_{\max }^{\prime}\right)
\end{aligned}
$$

All connections differ by an endomorphism-valued differential form of degree one, which can be viewed as a differential operator of degree zero. A differential operator of degree zero naturally extends to a bounded operator on the $L^{2}-$ Hilbert space, and hence does not pose additional restrictions on the domain. Therefore

$$
\mathcal{D}(\tilde{\nabla})=\mathcal{D}\left(\tilde{\nabla}^{\prime}\right), \quad \mathcal{D}\left(\widetilde{\nabla}^{*}\right)=\mathcal{D}\left(\left(\tilde{\nabla}^{\prime}\right)^{*}\right) .
$$

As a consequence of Lemma 3.3 we now directly obtain the following proposition.

Proposition 3.5 The chirality operator $\widetilde{\Gamma}$, restricted to the domains $\mathcal{D}(\widetilde{\nabla})$ and $\mathcal{D}\left(\widetilde{\nabla}^{*}\right)$, acts in the following way:

$$
\left.\widetilde{\Gamma}\right|_{\mathcal{D}(\tilde{\nabla})}: \mathcal{D}(\tilde{\nabla}) \rightarrow \mathcal{D}\left(\tilde{\nabla}^{*}\right),\left.\quad \widetilde{\Gamma}\right|_{\mathcal{D}\left(\tilde{\nabla}^{*}\right)}: \mathcal{D}\left(\tilde{\nabla}^{*}\right) \rightarrow \mathcal{D}(\tilde{\nabla})
$$

Moreover $\widetilde{\Gamma}$ relates the operators $\tilde{\nabla}$ and $\tilde{\nabla}^{\prime}$ as follows:

$$
\left.\widetilde{\Gamma}\right|_{\mathcal{D}(\widetilde{\nabla})} \tilde{\nabla}=\left.\left(\widetilde{\nabla}^{\prime}\right)^{*} \widetilde{\Gamma}\right|_{\mathcal{D}(\widetilde{\nabla} *)}
$$

Remark 3.6 The Fredholm complex $(\widetilde{\mathcal{D}}, \widetilde{\nabla})$, with the chirality operator $\widetilde{\Gamma}$ restricted to $\widetilde{\mathcal{D}}$, is in case of a flat Hermitian metric a complex with Poincare duality, in the sense of [9, Lemma 2.16], ie

$$
\nabla h^{E}=0 \Rightarrow \tilde{\Gamma} \widetilde{\nabla}=\widetilde{\nabla}^{*} \widetilde{\Gamma}
$$

We now apply the concepts of Braverman and Kappeler to our new setup.

Definition 3.7 The odd-signature operator of the Hilbert complex $(\widetilde{\mathcal{D}}, \widetilde{\nabla})$ is defined by

$$
\mathcal{B}:=\tilde{\Gamma} \tilde{\nabla}+\tilde{\nabla} \tilde{\Gamma}, \quad \mathcal{D}(\mathcal{B})=\mathcal{D}(\tilde{\nabla}) \cap \mathcal{D}\left(\tilde{\nabla}^{*}\right)
$$


Before we can state some basic properties of the odd signature operator, let us recall the notions of the Gauss-Bonnet operator and its relative and absolute self-adjoint extensions. The Gauss-Bonnet operator

$$
D^{\mathrm{GB}}:=\nabla+\nabla^{t}
$$

admits two natural self-adjoint extensions

$$
\begin{array}{ll}
D_{\text {rel }}^{\mathrm{GB}}=\nabla_{\text {min }}+\nabla_{\text {min }}^{*}, & \mathcal{D}\left(D_{\text {rel }}^{\mathrm{GB}}\right)=\mathcal{D}\left(\nabla_{\min }\right) \cap \mathcal{D}\left(\nabla_{\text {min }}^{*}\right), \\
D_{\text {abs }}^{\mathrm{GB}}=\nabla_{\max }+\nabla_{\text {max }}^{*}, & \mathcal{D}\left(D_{\mathrm{abs}}^{\mathrm{GB}}\right)=\mathcal{D}\left(\nabla_{\max }\right) \cap \mathcal{D}\left(\nabla_{\text {max }}^{*}\right),
\end{array}
$$

respectively called the relative and the absolute self-adjoint extensions. Their squares are correspondingly the relative and the absolute Laplace operators

$$
\triangle_{\text {rel }}=\left(D_{\text {rel }}^{\mathrm{GB}}\right)^{2}, \quad \triangle_{\mathrm{abs}}=\left(D_{\mathrm{abs}}^{\mathrm{GB}}\right)^{2},
$$

associated to the Hilbert complexes $\left(\mathcal{D}_{\min }, \nabla_{\min }\right)$ and $\left(\mathcal{D}_{\max }, \nabla_{\max }\right)$, respectively. Similar definitions, of course, hold for the Gauss-Bonnet operators $D_{\mathrm{rel}}^{\prime \mathrm{GB}}$ and $D_{\mathrm{abs}}^{\prime \mathrm{GB}}$ associated to the dual covariant derivative $\nabla^{\prime}$. Their squares

$$
\triangle_{\mathrm{rel}}^{\prime}=\left(D_{\mathrm{rel}}^{\prime \mathrm{GB}}\right)^{2}, \quad \triangle_{\mathrm{abs}}^{\prime}=\left(D_{\mathrm{abs}}^{\prime \mathrm{GB}}\right)^{2},
$$

are the Laplace operators, associated to the Hilbert complexes $\left(\mathcal{D}_{\min }^{\prime}, \nabla_{\min }^{\prime}\right)$ and $\left(\mathcal{D}_{\max }^{\prime}, \nabla_{\max }^{\prime}\right)$, respectively. Now we can state the following basic result.

Lemma 3.8 The leading symbols of $\mathcal{B}$ and $\tilde{\Gamma}\left(D_{\mathrm{rel}}^{\mathrm{GB}} \oplus D_{\mathrm{abs}}^{\prime \mathrm{GB}}\right)$ coincide and moreover

$$
\mathcal{D}(\mathcal{B})=\mathcal{D}\left(D_{\text {rel }}^{\mathrm{GB}} \oplus D_{\mathrm{abs}}^{\prime \mathrm{GB}}\right) .
$$

Proof First recall the relations

$$
\Gamma \nabla \Gamma=\left(\nabla^{\prime}\right)^{t}, \quad \Gamma \nabla^{t} \Gamma=\nabla^{\prime} .
$$

All connections differ by an endomorphism-valued differential form of degree one, which can be viewed as a differential operator of order zero. This implies the statement on the leading symbol of $\mathcal{B}$ and $\widetilde{\Gamma}\left(D^{\mathrm{GB}} \oplus D^{\prime \mathrm{GB}}\right)$

A differential operator of zero order naturally extends to a bounded operator on the $L^{2}$-Hilbert space, and hence does not pose additional restrictions on the domain, in particular we obtain (compare Lemma 3.3)

$$
\mathcal{D}\left(\nabla_{\min }^{*}\right)=\mathcal{D}\left(\Gamma \nabla_{\max } \Gamma\right), \quad \mathcal{D}\left(\nabla_{\max }^{*}\right)=\mathcal{D}\left(\Gamma \nabla_{\min } \Gamma\right) .
$$

Using these domain relations we find

$$
\mathcal{D}(\mathcal{B})=\mathcal{D}\left(\widetilde{\Gamma}\left(D_{\text {rel }}^{\mathrm{GB}} \oplus D_{\mathrm{abs}}^{\prime \mathrm{GB}}\right)\right)=\mathcal{D}\left(D_{\mathrm{rel}}^{\mathrm{GB}} \oplus D_{\mathrm{abs}}^{\prime \mathrm{GB}}\right) .
$$


Note by the arguments of the lemma above that $\mathcal{B}$ is a bounded perturbation of a closed operator $\widetilde{\Gamma}\left(D_{\mathrm{rel}}^{\mathrm{GB}} \oplus D_{\mathrm{abs}}^{\prime \mathrm{GB}}\right)$ and hence is closed, as well. Before we continue analyzing the spectral properties of the odd-signature operator $\mathcal{B}$, let us recall the following general result.

Theorem 3.9 (S Agmon, R Seeley) Let $\left(K, g^{K}\right)$ be a smooth compact oriented Riemannian manifold with boundary $\partial K$. Let $\left(F, h^{F}\right)$ be a Hermitian vector bundle over $K$. The metric structures $\left(g^{K}, h^{F}\right)$ define an $L^{2}$-inner product. Let

$$
D: C^{\infty}(K, F) \rightarrow C^{\infty}(K, F)
$$

be a differential operator of order $\omega$ such that $\omega \cdot \operatorname{rank} F$ is even. Consider a boundary value problem $(D, B)$ strongly elliptic with respect to $\mathbb{C} \backslash \mathbb{R}^{*}$ in the sense of [17]. Then:

(i) $D_{B}$ is a Fredholm operator with compact resolvent and discrete spectrum of eigenvalues of finite (algebraic) multiplicity, accumulating only at infinity.

(ii) The operator $D_{B}$ admits an Agmon angle $\theta \in(-\pi, 0)$ and the associated zetafunction

$$
\zeta\left(s, D_{B}\right):=\sum_{\lambda \in \operatorname{Spec}\left(D_{B}\right) \backslash\{0\}} m(\lambda) \cdot \lambda_{\theta}^{-s}, \quad \operatorname{Re}(s)>\frac{\operatorname{dim} K}{\omega},
$$

where $\lambda_{\theta}^{-s}:=\exp \left(-s \cdot \log _{\theta} \lambda\right)$ and $m(\lambda)$ denotes the multiplicity of the eigenvalue $\lambda$, is holomorphic for $\operatorname{Re}(s)>\operatorname{dim} K / \omega$ and admits a meromorphic extension to the whole complex plane $\mathbb{C}$ with $s=0$ being a regular point.

For the proof of the theorem note that the notion of strong ellipticity in the sense of [17] in fact combines ellipticity with Agmon's conditions, as in the treatment of elliptic boundary conditions by R Seeley in $[28 ; 27]$. The statement of the theorem above follows then from [1] and [28; 27]. This result is also stated and discussed by Burghelea, Friedlander and Kappeler in [11].

Remark 3.10 The definition of a zeta-function, as in Theorem 3.9 (ii), also applies to any operator $D$ with finite spectrum $\left\{\lambda_{1}, \ldots, \lambda_{n}\right\}$ and finite respective multiplicities $\left\{m_{1}, \ldots, m_{n}\right\}$. For a given Agmon angle $\theta \in[0,2 \pi)$ the associated zeta-function

$$
\zeta_{\theta}(s, D):=\sum_{i=1, \lambda_{i} \neq 0}^{n} m_{i} \cdot\left(\lambda_{i}\right)_{\theta}^{-s}
$$

is holomorphic for all $s \in \mathbb{C}$, since the sum is finite and the eigenvalue zero is excluded. 
Now we return to our specific setup. The following result is important in view of the relation between $\mathcal{B}$ and the Gauss-Bonnet operators with relative and absolute boundary conditions, as established in Lemma 3.8.

\section{Proposition 3.11 The operators}

$$
D=\widetilde{\Gamma}\left(D_{\mathrm{rel}}^{\mathrm{GB}} \oplus D_{\mathrm{abs}}^{\prime \mathrm{GB}}\right), \quad D^{2}=\triangle_{\mathrm{rel}} \oplus \triangle_{\mathrm{abs}}^{\prime}
$$

are strongly elliptic with respect to $\mathbb{C} \backslash \mathbb{R}^{*}$ and $\mathbb{C} \backslash \mathbb{R}^{+}$, respectively, in the sense of P Gilkey [17].

The fact that $D^{2}=\triangle_{\text {rel }} \oplus \triangle_{\text {rel }}^{\prime}$ is strongly elliptic with respect to $\mathbb{C} \backslash \mathbb{R}^{+}$is already encountered in Theorem 3.2. The strong ellipticity of $D$ now follows from [17, Lemma 1.11.2]. Note that this result in the reference [17] is proved explicitly, even though other aspects of [17, Section 1.11] are rather expository.

Since Lemma 3.8 asserts the equality between the leading symbols of the differential operators $\mathcal{B}, D$ and moreover the equality of the associated boundary conditions, the odd signature operator $\mathcal{B}$ and its square $\mathcal{B}^{2}$ are strongly elliptic as well. This proves together with Theorem 3.9 the next proposition.

Proposition 3.12 The operators $\mathcal{B}$ and $\mathcal{B}^{2}$ are strongly elliptic with respect to $\mathbb{C} \backslash \mathbb{R}^{*}$ and $\mathbb{C} \backslash \mathbb{R}^{+}$, respectively, in the sense of PGilkey [17]. The operators $\mathcal{B}, \mathcal{B}^{2}$ have discrete spectrum accumulating only at infinity.

Let now $\lambda \geq 0$ be any nonnegative real number. Denote by $\Pi_{\mathcal{B}^{2},[0, \lambda]}$ the spectral projection of $\mathcal{B}^{2}$ onto eigenspaces with eigenvalues of absolute value in the interval $[0, \lambda]$ :

$$
\Pi_{\mathcal{B}^{2},[0, \lambda]}:=\frac{i}{2 \pi} \int_{C(\lambda)}\left(\mathcal{B}^{2}-x\right)^{-1} d x
$$

with $C(\lambda)$ being any closed counterclockwise circle surrounding eigenvalues of absolute value in $[0, \lambda]$ with no other eigenvalue inside. One finds using the analytic Fredholm theorem that the range of the projection lies in the domain of $\mathcal{B}^{2}$ and that the projection commutes with $\mathcal{B}^{2}$.

Since $\mathcal{B}^{2}$ has discrete spectrum accumulating only at infinity, the spectral projection $\Pi_{\mathcal{B}^{2},[0, \lambda]}$ is of finite rank, ie with a finite-dimensional image. In particular $\Pi_{\mathcal{B}^{2},[0, \lambda]}$ is a bounded operator in $L_{*}^{2}(M, E \oplus E)$. Hence with [21, Section 4, page 155] the decomposition

$$
L_{*}^{2}(M, E \oplus E)=\text { Image } \Pi_{\mathcal{B}^{2},[0, \lambda]} \oplus \operatorname{Image}\left(1-\Pi_{\mathcal{B}^{2},[0, \lambda]}\right),
$$

is a direct sum decomposition into closed subspaces of the Hilbert space $L_{*}^{2}(M, E \oplus E)$. 
Note that if $\mathcal{B}^{2}$ is self-adjoint, the decomposition is orthogonal with respect to the fixed $L^{2}$-Hilbert structure, ie the projection $\Pi_{\mathcal{B}^{2},[0, \lambda]}$ is an orthogonal projection, which is the case only if the Hermitian metric $h^{E}$ is flat with respect to $\nabla$.

Since $\mathcal{D}\left(\mathcal{B}^{2}\right) \subset \widetilde{\mathcal{D}}$, where $\widetilde{\mathcal{D}}$ was introduced in Definition 3.4, decomposition (3-5) induces a decomposition of $\widetilde{\mathcal{D}}$ into nontrivial direct sum components:

$$
\widetilde{\mathcal{D}}=\widetilde{\mathcal{D}}_{[0, \lambda]} \oplus \widetilde{\mathcal{D}}_{(\lambda, \infty)}
$$

Since $\tilde{\nabla}$ commutes with $\mathcal{B}, \mathcal{B}^{2}$ and hence also with $\Pi_{\mathcal{B}^{2},[0, \lambda]}$, we find that the decomposition above is in fact a decomposition into subcomplexes:

$$
(\tilde{\mathcal{D}}, \tilde{\nabla})=\left(\widetilde{\mathcal{D}}_{[0, \lambda]}, \tilde{\nabla}_{[0, \lambda]}\right) \oplus\left(\tilde{\mathcal{D}}_{(\lambda, \infty)}, \widetilde{\nabla}_{(\lambda, \infty)}\right)
$$

where

$$
\widetilde{\nabla}_{\mathcal{I}}:=\left.\widetilde{\nabla}\right|_{\widetilde{\mathcal{D}}_{\mathcal{I}}} \text { for } \mathcal{I}=[0, \lambda] \text { or }(\lambda, \infty) \text {. }
$$

Further $\widetilde{\Gamma}$ also commutes with $\mathcal{B}, \mathcal{B}^{2}$ and hence also with $\Pi_{\mathcal{B}^{2},[0, \lambda]}$. Thus as above we obtain

$$
\widetilde{\Gamma}=\widetilde{\Gamma}_{[0, \lambda]} \oplus \widetilde{\Gamma}_{(\lambda, \infty)} .
$$

Consequently the odd-signature operator of the complex $(\tilde{\mathcal{D}}, \widetilde{\nabla})$ decomposes correspondingly:

$$
\mathcal{B}=\mathcal{B}^{[0, \lambda]} \oplus \mathcal{B}^{(\lambda, \infty)}
$$

where

$$
\mathcal{B}^{\mathcal{I}}:=\widetilde{\Gamma}_{\mathcal{I}} \tilde{\nabla}_{\mathcal{I}}+\widetilde{\nabla}_{\mathcal{I}} \widetilde{\Gamma}_{\mathcal{I}} \text { for } \mathcal{I}=[0, \lambda] \text { or }(\lambda, \infty) .
$$

The closedness of the subspace Image $\left(1-\Pi_{\mathcal{B}^{2},[0, \lambda]}\right)$ implies that the domain of $\mathcal{B}^{(\lambda, \infty)}$

$$
\mathcal{D}\left(\mathcal{B}^{(\lambda, \infty)}\right):=\mathcal{D}(\mathcal{B}) \cap \operatorname{Image}\left(1-\Pi_{\mathcal{B}^{2},[0, \lambda]}\right)
$$

is closed under the graph-norm, hence the operator $\mathcal{B}^{(\lambda, \infty)}$ is a closed operator in the Hilbert space Image $\left(1-\Pi_{\mathcal{B}^{2},[0, \lambda]}\right)$.

We need to analyze the direct sum component $\mathcal{B}^{(\lambda, \infty)}$. For this we proceed with the following general functional analytic observations.

Proposition 3.13 Let $D$ be a closed operator in a separable Hilbert space $(H,\langle\cdot, \cdot\rangle)$. The domain $\mathcal{D}(D)$ is a Hilbert space with the graph-norm

$$
\langle x, y\rangle_{D}=\langle x, y\rangle+\langle D x, D y\rangle
$$

for any $x, y \in \mathcal{D}(D)$. Let $\operatorname{Res} D \neq \varnothing$. Then the following statements are equivalent:

(1) The inclusion $\iota: \mathcal{D}(D) \hookrightarrow H$ is a compact operator.

(2) $D$ has a compact resolvent, ie for some (and thus for all) $z \in \operatorname{Res}(D)$ the resolvent operator $(D-z)^{-1}$ is a compact operator on $H$. 
Proof Assume first that the inclusion $\iota: \mathcal{D}(D) \hookrightarrow H$ is a compact operator. Since Spec $D \neq \mathbb{C}$ the resolvent set $\operatorname{Res}(D)$ is not empty. For any $z \in \operatorname{Res}(D)$ the resolvent operator

$$
(D-z)^{-1}: H \rightarrow \mathcal{D}(D)
$$

exists and is bounded, by definition of the resolvent set. With the inclusion $\iota$ being a compact operator we find directly that $(D-z)^{-1}$ is compact as an operator from $H$ to $H$. Finally, if $(D-z)^{-1}$ is compact for some $z \in \operatorname{Res}(D)$, then by the second resolvent identity it is compact for all $z \in \operatorname{Res}(D)$; see also [21, page 187].

Conversely assume that for some (and therefore for all) $z \in \operatorname{Res}(D)$ the resolvent operator $(D-z)^{-1}$ is compact as an operator from $H$ into $H$. Observe

$$
\iota=(D-z)^{-1} \circ(D-z): \mathcal{D}(D) \hookrightarrow H .
$$

By compactness of the resolvent operator, $\iota$ is compact as an operator between the Hilbert spaces $\mathcal{D}(D)$ and $H$.

Proposition 3.14 Let $D$ be a closed operator in a separable Hilbert space $H$ with $\operatorname{Res}(D) \neq \varnothing$ and compact resolvent. Then $D$ is a Fredholm operator with

$$
\text { index } D=0 \text {. }
$$

Proof By closedness of $D$ the domain $\mathcal{D}(D)$ turns into a Hilbert space equipped with the graph norm. By Proposition 3.13 the natural inclusion

$$
\iota: \mathcal{D}(D) \hookrightarrow H
$$

is a compact operator. Therefore, viewing $\mathcal{D}(D)$ as a subspace of $H$, ie endowed with the inner product of $H$, the inclusion

$$
\iota: \mathcal{D}(D) \subset H \hookrightarrow H
$$

is relatively $D$-compact in the sense of [21, Section 4.3, page 194]. More precisely this means, that if for a sequence $\left\{u_{n}\right\} \subset \mathcal{D}(D)$ both $\left\{u_{n}\right\}$ and $\left\{D u_{n}\right\}$ are bounded sequences in $H$, then $\left\{\iota\left(u_{n}\right)\right\} \subset H$ has a convergent subsequence.

Now for any $\lambda \in \mathbb{C} \backslash \operatorname{Spec}(D)$ the operator

$$
(D-\lambda \iota): \mathcal{D}(D) \subset H \rightarrow H
$$

is invertible and hence trivially a Fredholm operator with trivial kernel and closed range $H$. In particular

$$
\operatorname{index}(D-\lambda \iota)=0
$$


Now, from stability of the Fredholm index under relatively compact perturbations (see Kato [21, Theorem 5.26] and the references therein) we infer with the inclusion $\iota$ being relatively compact, that $D$ is a Fredholm operator of zero index:

$$
\text { index } D=\operatorname{index}(D-\lambda \iota)=0 \text {. }
$$

Corollary 3.15 The operator $\mathcal{B}^{(\lambda, \infty)}: \mathcal{D}\left(\mathcal{B}^{(\lambda, \infty)}\right) \rightarrow \operatorname{Image}\left(1-\Pi_{\mathcal{B}^{2},[0, \lambda]}\right)$ of the complex $\left(\widetilde{\mathcal{D}}_{(\lambda, \infty)}, \widetilde{\nabla}_{(\lambda, \infty)}\right)$ with $\lambda \geq 0$ is bijective.

Proof Consider any $\lambda \in \mathbb{C} \backslash \operatorname{Spec} \mathcal{B}$. By the strong ellipticity of $\mathcal{B}$, the operator

$$
(\mathcal{B}-\lambda): \mathcal{D}(\mathcal{B}) \rightarrow L_{*}^{2}(M, E \oplus E)
$$

is bijective with compact inverse. Hence we immediately find that the restriction

$$
\left(\mathcal{B}^{(\lambda, \infty)}-\lambda\right) \equiv(\mathcal{B}-\lambda) \uparrow \operatorname{Im}\left(1-\Pi_{\mathcal{B}^{2},[0, \lambda]}\right): \mathcal{D}\left(\mathcal{B}^{(\lambda, \infty)}\right) \rightarrow \operatorname{Im}\left(1-\Pi_{\mathcal{B}^{2},[0, \lambda]}\right)
$$

is bijective with compact inverse, as well. Now we deduce from Proposition 3.14 that $\mathcal{B}^{(\lambda, \infty)}$ is Fredholm with

$$
\operatorname{index} \mathcal{B}^{(\lambda, \infty)}=0 .
$$

The operator $\mathcal{B}^{(\lambda, \infty)}$ is injective, by definition. Combining injectivity with the vanishing of the index, we derive surjectivity of $\mathcal{B}^{(\lambda, \infty)}$. This proves the statement.

Note, that in case of a flat Hermitian metric the assertion of the previous corollary is simply the general fact that a self-adjoint Fredholm operator is invertible if and only if its kernel is trivial.

Corollary 3.16 The subcomplex $\left(\widetilde{\mathcal{D}}_{(\lambda, \infty)}, \widetilde{\nabla}_{(\lambda, \infty)}\right)$ is acyclic and

$$
H^{*}\left(\left(\tilde{\mathcal{D}}_{[0, \lambda]}, \tilde{\nabla}_{[0, \lambda]}\right)\right) \cong H^{*}(\tilde{\mathcal{D}}, \tilde{\nabla}) .
$$

Proof Corollary 3.15 allows us to apply the purely algebraic result [6, Lemma 5.8]. Consequently $\left(\widetilde{\mathcal{D}}_{(\lambda, \infty)}, \widetilde{\nabla}_{(\lambda, \infty)}\right)$ is an acyclic complex. Together with the decomposition (3-6) this proves the assertion.

Since the spectrum of $\mathcal{B}^{2}$ is discrete accumulating only at infinity, $\left(\widetilde{\mathcal{D}}_{[0, \lambda]}, \widetilde{\nabla}_{[0, \lambda]}\right)$ is a complex of finite-dimensional complex vector spaces where $\widetilde{\Gamma}_{[0, \lambda]}: \widetilde{\mathcal{D}}_{[0, \lambda]}^{k} \rightarrow \widetilde{\mathcal{D}}_{[0, \lambda]}^{m-k}$ is the chirality operator on the complex in the sense of [6, Section 1.1].

We also use the notion of determinant lines of finite dimensional complexes, going back at least to [22], which are given for any finite complex of finite-dimensional vector spaces $\left(C^{*}, \partial_{*}\right)$ as follows:

$$
\operatorname{Det} H^{*}\left(C^{*}, \partial_{*}\right)=\bigotimes_{k} \operatorname{det} H^{k}\left(C^{*}, \partial_{*}\right)^{(-1)^{k}}
$$


where the determinant $\operatorname{det} H^{k}\left(C^{*}, \partial_{*}\right)$ is the top exterior power of $H^{k}\left(C^{*}, \partial_{*}\right)$ and $\operatorname{det} H^{k}\left(C^{*}, \partial_{*}\right)^{-1} \equiv \operatorname{det} H^{k}\left(C^{*}, \partial_{*}\right)^{*}$. The notion of determinant lines of finite dimensional complexes is also discussed in [6]. We follow [6, Section 1.1] and form the "refined torsion" (note the difference from "refined analytic torsion") of the complex $\left(\widetilde{\mathcal{D}}_{[0, \lambda]}, \widetilde{\nabla}_{[0, \lambda]}\right)$ :

$$
\begin{aligned}
\rho_{[0, \lambda]}:=c_{0} \otimes\left(c_{1}\right)^{-1} \otimes \cdots \otimes\left(c_{r}\right)^{(-1)^{r}} \otimes\left(\widetilde{\Gamma}_{[0, \lambda]} c_{r}\right)^{(-1)^{r+1}} & \\
& \otimes \cdots \otimes\left(\widetilde{\Gamma}_{[0, \lambda]} c_{1}\right) \otimes\left(\widetilde{\Gamma}_{[0, \lambda]} c_{0}\right)^{(-1)} \in \operatorname{Det}\left(H^{*}\left(\widetilde{\mathcal{D}}_{[0, \lambda]}, \widetilde{\nabla}_{[0, \lambda]}\right)\right),
\end{aligned}
$$

where $c_{k} \in \operatorname{det} H^{k}\left(\widetilde{\mathcal{D}}_{[0, \lambda]}, \widetilde{\nabla}_{[0, \lambda]}\right)$ are arbitrary elements of the determinant lines, $\widetilde{\Gamma}_{[0, \lambda]}$ denotes the chirality operator

$$
\widetilde{\Gamma}_{[0, \lambda]}: \widetilde{\mathcal{D}}_{[0, \lambda]}^{\bullet} \rightarrow \widetilde{\mathcal{D}}_{[0, \lambda]}^{m-\bullet}
$$

extended to determinant lines and for any $v \in \operatorname{det} H^{k}\left(\widetilde{\mathcal{D}}_{[0, \lambda]}, \widetilde{\nabla}_{[0, \lambda]}\right)$ the dual $v^{-1} \in$ $\operatorname{det} H^{k}\left(\widetilde{\mathcal{D}}_{[0, \lambda]}, \widetilde{\nabla}_{[0, \lambda]}\right)^{-1} \equiv \operatorname{det} H^{k}\left(\widetilde{\mathcal{D}}_{[0, \lambda]}, \widetilde{\nabla}_{[0, \lambda]}\right)^{*}$ is the unique element such that $v^{-1}(v)=1$.

By Corollary 3.16 we can view $\rho_{[0, \lambda]}$ canonically as an element of $\operatorname{Det}\left(H^{*}(\widetilde{\mathcal{D}}, \widetilde{\nabla})\right)$, which we do henceforth.

The second part of the construction is the graded determinant. The operator $\mathcal{B}^{(\lambda, \infty)}$, $\lambda \geq 0$ is bijective by Corollary 3.15 and hence by injectivity (put $\mathcal{I}=(\lambda, \infty)$ to simplify the notation)

$$
\operatorname{ker}\left(\widetilde{\nabla}_{\mathcal{I}} \widetilde{\Gamma}_{\mathcal{I}}\right) \cap \operatorname{ker}\left(\widetilde{\Gamma}_{\mathcal{I}} \widetilde{\nabla}_{\mathcal{I}}\right)=\{0\} .
$$

Further the complex $\left(\widetilde{\mathcal{D}}_{\mathcal{I}}, \widetilde{\nabla}_{\mathcal{I}}\right)$ is acyclic by Corollary 3.16 and due to $\widetilde{\Gamma}_{\mathcal{I}}$ being an involution on $\operatorname{Im}\left(1-\Pi_{\mathcal{B}^{2},[0, \lambda]}\right)$ we have

$$
\begin{aligned}
& \operatorname{ker}\left(\tilde{\nabla}_{\mathcal{I}} \widetilde{\Gamma}_{\mathcal{I}}\right)=\widetilde{\Gamma}_{\mathcal{I}} \operatorname{ker}\left(\tilde{\nabla}_{\mathcal{I}}\right)=\widetilde{\Gamma}_{\mathcal{I}} \operatorname{Im}\left(\widetilde{\nabla}_{\mathcal{I}}\right)=\operatorname{Im}\left(\widetilde{\Gamma}_{\mathcal{I}} \widetilde{\nabla}_{\mathcal{I}}\right), \\
& \operatorname{ker}\left(\widetilde{\Gamma}_{\mathcal{I}} \tilde{\nabla}_{\mathcal{I}}\right)=\operatorname{ker}\left(\widetilde{\nabla}_{\mathcal{I}}\right)=\operatorname{Im}\left(\widetilde{\nabla}_{\mathcal{I}}\right)=\operatorname{Im}\left(\widetilde{\nabla}_{\mathcal{I}} \widetilde{\Gamma}_{\mathcal{I}}\right) .
\end{aligned}
$$

We have $\operatorname{Im}\left(\widetilde{\Gamma}_{\mathcal{I}} \widetilde{\nabla}_{\mathcal{I}}\right)+\operatorname{Im}\left(\widetilde{\nabla}_{\mathcal{I}} \widetilde{\Gamma}_{\mathcal{I}}\right)=\operatorname{Im}\left(\mathcal{B}^{\mathcal{I}}\right)$ and by surjectivity of $\mathcal{B}^{\mathcal{I}}$ we obtain from the last three relations above

$$
\operatorname{Im}\left(1-\Pi_{\mathcal{B}^{2},[0, \lambda]}\right)=\operatorname{ker}\left(\widetilde{\nabla}_{\mathcal{I}} \widetilde{\Gamma}_{\mathcal{I}}\right) \oplus \operatorname{ker}\left(\widetilde{\Gamma}_{\mathcal{I}} \widetilde{\nabla}_{\mathcal{I}}\right) .
$$

Note that $\mathcal{B}$ leaves $\operatorname{ker}(\tilde{\nabla} \widetilde{\Gamma})$ and $\operatorname{ker}(\tilde{\Gamma} \widetilde{\nabla})$ invariant. Put

$$
\begin{aligned}
& \mathcal{B}_{\text {even }}^{+,(\lambda, \infty)}:=\mathcal{B}^{(\lambda, \infty)} \uparrow \tilde{\mathcal{D}}^{\text {even }} \cap \operatorname{ker}(\tilde{\nabla} \widetilde{\Gamma}), \\
& \mathcal{B}_{\text {even }}^{-,(\lambda, \infty)}:=\mathcal{B}^{(\lambda, \infty)} \uparrow \tilde{\mathcal{D}}^{\text {even }} \cap \operatorname{ker}(\tilde{\Gamma} \widetilde{\nabla}) .
\end{aligned}
$$


We obtain a direct sum decomposition

$$
\mathcal{B}_{\text {even }}^{(\lambda, \infty)}=\mathcal{B}_{\text {even }}^{+,(\lambda, \infty)} \oplus \mathcal{B}_{\text {even }}^{-,(\lambda, \infty)} .
$$

As a consequence of Theorem 3.9 (ii) and Proposition 3.12 there exists an Agmon angle $\theta \in(-\pi, 0)$ for $\mathcal{B}$, which is clearly an Agmon angle for the restrictions above, as well.

By Theorem 3.9 and Proposition 3.12 the zeta function $\zeta_{\theta}(s, \mathcal{B})$ is holomorphic for $\operatorname{Re}(s)$ sufficiently large. The zeta-functions $\zeta_{\theta}\left(s, \mathcal{B}_{\text {even }}^{ \pm,(\lambda, \infty)}\right)$ of $\mathcal{B}_{\text {even }}^{ \pm,(\lambda, \infty)}$, defined with respect to the given Agmon angle $\theta$, are holomorphic for $\operatorname{Re}(s)$ large as well, since the restricted operators have the same spectrum as $\mathcal{B}$ but in general with lower or at most the same multiplicities.

We define the graded zeta-function

$$
\zeta_{\mathrm{gr}, \theta}\left(s, \mathcal{B}_{\text {even }}^{(\lambda, \infty)}\right):=\zeta_{\theta}\left(s, \mathcal{B}_{\text {even }}^{+,(\lambda, \infty)}\right)-\zeta_{\theta}\left(s,-\mathcal{B}_{\text {even }}^{-,(\lambda, \infty)}\right), \operatorname{Re}(s) \gg 0 .
$$

In the next subsection we prove in Theorem 4.4 that the graded zeta-function extends meromorphically to $\mathbb{C}$ and is regular at $s=0$. For the time being we shall assume regularity at zero and define the graded determinant.

Definition 3.17 (Graded determinant) Let $\theta \in(-\pi, 0)$ be an Agmon angle for $\mathcal{B}^{(\lambda, \infty)}$. Then the "graded determinant" associated to $\mathcal{B}^{(\lambda, \infty)}$ and its Agmon angle $\theta$ is defined as follows:

$$
\operatorname{det}_{\mathrm{gr}, \theta}\left(\mathcal{B}_{\text {even }}^{(\lambda, \infty)}\right):=\exp \left(-\left.\frac{d}{d s}\right|_{s=0} \zeta_{\mathrm{gr}, \theta}\left(s, \mathcal{B}_{\text {even }}^{(\lambda, \infty)}\right)\right) .
$$

\section{Proposition 3.18 The element}

$$
\rho\left(\nabla, g^{M}\right):=\operatorname{det}_{\mathrm{gr}, \theta}\left(\mathcal{B}_{\text {even }}^{(\lambda, \infty)}\right) \cdot \rho_{[0, \lambda]} \in \operatorname{Det}\left(H^{*}(\tilde{\mathcal{D}}, \tilde{\nabla})\right)
$$

is independent of the choice of $\lambda \geq 0$ and choice of Agmon angle $\theta \in(-\pi, 0)$ for the odd-signature operator $\mathcal{B}^{(\lambda, \infty)}$.

Proof Let $0 \leq \lambda<\mu<\infty$. We obtain $\widetilde{\mathcal{D}}_{[0, \mu]}=\widetilde{\mathcal{D}}_{[0, \lambda]} \oplus \widetilde{\mathcal{D}}_{(\lambda, \mu]}$ and also $\widetilde{\mathcal{D}}_{(\lambda, \infty)}=$ $\widetilde{\mathcal{D}}_{(\lambda, \mu]} \oplus \widetilde{\mathcal{D}}_{(\mu, \infty)}$. Since the odd-signature operator respects this spectral direct sum decomposition (see (3-7)), we obtain

$$
\operatorname{det}_{\mathrm{gr}}\left(\mathcal{B}_{\text {even }}^{(\lambda, \infty)}\right)=\operatorname{det}_{\mathrm{gr}}\left(\mathcal{B}_{\text {even }}^{(\mu, \infty)}\right) \cdot \operatorname{det}_{\mathrm{gr}}\left(\mathcal{B}_{\text {even }}^{(\lambda, \mu]}\right) .
$$

Further the purely algebraic discussion behind [6, Proposition 5.10] implies

$$
\rho_{[0, \mu]}=\operatorname{det}_{\mathrm{gr}}\left(\mathcal{B}_{\text {even }}^{(\lambda, \mu]}\right) \cdot \rho_{[0, \lambda]} .
$$


This proves the equality

$$
\operatorname{det}_{\mathrm{gr}}\left(\mathcal{B}_{\text {even }}^{(\lambda, \infty)}\right) \cdot \rho_{[0, \lambda]}=\operatorname{det}_{\mathrm{gr}}\left(\mathcal{B}_{\text {even }}^{(\mu, \infty)}\right) \cdot \rho_{[0, \mu]} .
$$

To see independence of $\theta \in(-\pi, 0)$ note that the strongly elliptic operator (cf Lemma $3.8)$

$$
D:=\widetilde{\Gamma}\left(D_{\mathrm{rel}}^{\mathrm{GB}} \oplus D_{\mathrm{abs}}^{\prime \mathrm{GB}}\right)
$$

is self-adjoint and $\mathcal{B}$ differs from $D$ by a bounded perturbation. By a Neumann-series argument and the asymptotics of the resolvent for $D$ (see Seeley [28, Lemma 15]) we get:

$$
\forall \theta \in(-\pi, 0): \quad \operatorname{Spec}(\mathcal{B}) \cap R_{\theta} \text { is finite. }
$$

By discreteness of $\operatorname{Spec}(\mathcal{B})$ we deduce that if $\theta, \theta^{\prime} \in(-\pi, 0)$ are both Agmon angles for $\mathcal{B}^{(\lambda, \infty)}$, there are only finitely many eigenvalues of $\mathcal{B}^{(\lambda, \infty)}$ in the solid angle between $\theta$ and $\theta^{\prime}$. Hence

$$
\left.\left.\left.\frac{d}{d s}\right|_{s=0} \zeta_{\mathrm{gr}, \theta}\left(s, \mathcal{B}_{\mathrm{even}}^{(\lambda, \infty)}\right)\right)\left.\equiv \frac{d}{d s}\right|_{s=0} \zeta_{\mathrm{gr}, \theta^{\prime}}\left(s, \mathcal{B}_{\text {even }}^{(\lambda, \infty)}\right)\right) \bmod 2 \pi i,
$$

and therefore

$$
\operatorname{det}_{\mathrm{gr}, \theta}\left(\mathcal{B}_{\text {even }}^{(\lambda, \infty)}\right)=\operatorname{det}_{\mathrm{gr}, \theta^{\prime}}\left(\mathcal{B}_{\text {even }}^{(\lambda, \infty)}\right) .
$$

This proves independence of the choice of $\theta \in(-\pi, 0)$ and completes the proof.

Note that the construction of $\rho\left(\nabla, g^{M}\right)$ is in fact independent of the choice of a Hermitian metric $h^{E}$. Indeed, a variation of $h^{E}$ does not change the odd-signature operator $\mathcal{B}$ as a differential operator. However it enters a priori the definition of $\mathcal{D}(\mathcal{B})$, since $h^{E}$ defines the $L^{2}-$ Hilbert space.

Nevertheless different Hermitian metrics give rise to equivalent $L^{2}$-norms over compact manifolds. Hence a posteriori the domain $\mathcal{D}(\mathcal{B})$ is indeed independent of the particular choice of $h^{E}$. Independence of the choice of a Hermitian metric $h^{E}$ is essential, since for nonunitary flat vector bundles there is no canonical choice of $h^{E}$ and Hermitian metric is fixed arbitrarily.

In the next section we determine the dependence of $\rho\left(\nabla, g^{M}\right)$ on the Riemannian metric $g^{M}$, in order to construct a differential invariant, which will be called the refined analytic torsion.

\section{Metric anomaly and refined analytic torsion}

We introduce the notion of eta-function leading to the notion of the eta-invariant of an elliptic operator. The eta-invariant was first introduced by Atiyah, Patodi and Singer in [2] as the boundary correction term in their index formula. 
Theorem 4.1 (P B Gilkey-L Smith) Let $\left(K, g^{K}\right)$ be a smooth compact oriented Riemannian manifold with boundary $\partial K$. Let $\left(F, h^{F}\right)$ be a Hermitian vector bundle and let the metric structures $\left(g^{K}, h^{F}\right)$ define an $L^{2}-$ scalar product. Let

$$
D: C^{\infty}(K, F) \rightarrow C^{\infty}(K, F)
$$

be a differential operator of order $\omega$ such that $\omega \cdot \operatorname{rank} F$ is even. Let a boundary value problem $(D, B)$ be strongly elliptic with respect to $\mathbb{C} \backslash \mathbb{R}^{*}$ and an Agmon angle $\theta \in(-\pi, 0)$. Then we have:

(i) $D_{B}$ is a Fredholm operator in the Hilbert space $L^{2}(K, F)$ with discrete spectrum and its eta-function

$$
\eta_{\theta}\left(s, D_{B}\right):=\sum_{\operatorname{Re}(\lambda)>0} m(\lambda) \cdot \lambda_{\theta}^{-s}-\sum_{\operatorname{Re}(\lambda)<0} m(\lambda) \cdot(-\lambda)_{\theta}^{-s},
$$

where $m(\lambda)$ denotes the finite (algebraic) multiplicity of the eigenvalue $\lambda$, is holomorphic for $\operatorname{Re}(s)$ large and extends meromorphically to $\mathbb{C}$ with at most simple poles.

(ii) If $D$ is of order one with the leading symbol $\sigma_{D}(x, \xi), x \in K, \xi \in T_{x}^{*} K$ satisfying

$$
\sigma_{D}(x, \xi)^{2}=|\xi|^{2} \cdot I,
$$

where $I$ is $\operatorname{rank} F \times \operatorname{rank} F$ identity matrix, and the boundary condition $B$ is of order zero, then the meromorphic extension of $\eta_{\theta}\left(s, D_{B}\right)$ is regular at $s=0$.

The proof of the theorem follows from the results in $[18 ; 19]$ on the eta-function of strongly elliptic boundary value problems. The fact that $\eta_{\theta}\left(s, D_{B}\right)$ is holomorphic for $\operatorname{Re}(s)$ sufficiently large is asserted in [18, Lemma 2.3 (c)]. The meromorphic continuation with at most isolated simple poles is asserted in [18, Theorem 2.7].

The fact that $s=0$ is a regular point of the eta-function is highly nontrivial and cannot be proved by local arguments. Using homotopy invariance of the residue at zero for the eta-function, P B Gilkey and L Smith [19] reduced the discussion to a certain class of operators with constant coefficients in the collar neighborhood of the boundary and applied the closed double manifold argument. The reduction works for differential operators of order one with 0 -th order boundary conditions under the assumption on the leading symbol of the operator as in the second statement of the theorem. The regularity statement of Theorem 4.1 follows directly from [19, Theorem 2.3.5] and [19, Lemma 2.3.4].

Remark 4.2 The definition of an eta-function, as in Theorem $4.1(i)$, also applies to any operator $D$ with finite spectrum $\left\{\lambda_{1}, \ldots, \lambda_{n}\right\}$ and finite respective multiplicities 
$\left\{m_{1}, \ldots, m_{n}\right\}$. For a given Agmon angle $\theta \in[0,2 \pi)$ the associated eta-function

$$
\eta_{\theta}(s, D):=\sum_{\operatorname{Re}(\lambda)>0} m(\lambda) \cdot \lambda_{\theta}^{-s}-\sum_{\operatorname{Re}(\lambda)<0} m(\lambda) \cdot(-\lambda)_{\theta}^{-s},
$$

is holomorphic for all $s \in \mathbb{C}$, since the sum is finite and the zero-eigenvalue is excluded.

Proposition 4.3 The eta-function $\eta_{\theta}\left(s, \mathcal{B}_{\text {even }}\right)$ associated to the even part $\mathcal{B}_{\text {even }}$ of the odd-signature operator and its Agmon angle $\theta \in(-\pi, 0)$, is holomorphic for $\operatorname{Re}(s)$ large and extends meromorphically to $\mathbb{C}$ with $s=0$ being a regular point.

The statement of the proposition on the meromorphic extension of the eta-function is a direct consequence of Theorem 4.1 (i) and Proposition 3.12. The regularity statement follows from Theorem 4.1 (ii) and an explicit computation of the leading symbol of the odd-signature operator; compare also [19, Example 2.2.4].

Using Proposition 4.3 we can define the eta-invariant in the manner of [6] for $\mathcal{B}_{\text {even }}$ :

$$
\eta\left(\mathcal{B}_{\text {even }}\right):=\frac{1}{2}\left(\eta_{\theta}\left(s=0, \mathcal{B}_{\text {even }}\right)+m_{+}-m_{-}+m_{0}\right),
$$

where $m_{ \pm}$is the number of $\mathcal{B}_{\text {even }}$ - eigenvalues on the positive, respectively the negative part of the imaginary axis and $m_{0}$ is the dimension of the generalized zero-eigenspace of $\mathcal{B}_{\text {even }}$.

Implicit in the notation is also the fact, that $\eta\left(\mathcal{B}_{\text {even }}\right)$ does not depend on the Agmon angle $\theta \in(-\pi, 0)$. This is due to the fact that, given a different Agmon angle $\theta^{\prime} \in$ $(-\pi, 0)$, there are by $(3-13)$ and discreteness of $\operatorname{Spec}(\mathcal{B})$ only finitely many eigenvalues of $\mathcal{B}_{\text {even }}$ in the acute angle between $\theta$ and $\theta^{\prime}$.

Similarly we define the eta-invariants of $\mathcal{B}_{\text {even }}^{(\lambda, \infty)}$ and $\mathcal{B}_{\text {even }}^{[0, \lambda]}$ and in particular we get

$$
\eta\left(\mathcal{B}_{\text {even }}\right)=\eta\left(\mathcal{B}_{\text {even }}^{(\lambda, \infty)}\right)+\eta\left(\mathcal{B}_{\text {even }}^{[0, \lambda]}\right)
$$

Before we prove the next central result, let us make the following observation.

Consider the imaginary axis $i \mathbb{R} \subset \mathbb{C}$. By (3-13) there are only finitely many eigenvalues of $\mathcal{B}$ on $i \mathbb{R}$. Further by the discreteness of $\operatorname{Spec}(\mathcal{B})$ small rotation of the imaginary axis does not hit any further eigenvalue of $\mathcal{B}$ and in particular of $\mathcal{B}_{\text {even }}^{(\lambda, \infty)}, \lambda \geq 0$. More precisely this means that there exists an $\epsilon>0$ sufficiently small such that the angle

$$
\theta:=-\frac{\pi}{2}+\epsilon
$$


is an Agmon angle for $\mathcal{B}_{\text {even }}^{(\lambda, \infty)}$ and the solid angles

$$
\begin{aligned}
L_{(-\pi / 2, \theta]} & :=\left\{z \in \mathbb{C}|z=| z \mid \cdot e^{i \phi}, \phi \in(-\pi / 2, \theta]\right\}, \\
L_{(\pi / 2, \theta+\pi]} & :=\left\{z \in \mathbb{C}|z=| z \mid \cdot e^{i \phi}, \phi \in(\pi / 2, \theta+\pi]\right\}
\end{aligned}
$$

do not contain eigenvalues of $\mathcal{B}_{\text {even }}^{(\lambda, \infty)}$. With this observation we can state the following central result:

Theorem 4.4 Let $\theta \in(-\pi / 2,0)$ be an Agmon angle for $\mathcal{B}_{\text {even }}^{(\lambda, \infty)}$ such that there are no eigenvalues of $\mathcal{B}_{\text {even }}^{(\lambda, \infty)}$ in the solid angles $L_{(-\pi / 2, \theta]}$ and $L_{(-\pi / 2, \theta+\pi]}$. Then $2 \theta$ is an Agmon angle for $\left(\mathcal{B}_{\text {even }}^{(\lambda, \infty)}\right)^{2}$ and hence for $\mathcal{B}^{2} \backslash \widetilde{\mathcal{D}}_{(\lambda, \infty)}^{k}$ in each degree $k=0, \ldots, \operatorname{dim} M$. Then the graded zeta-function $\zeta_{\mathrm{gr}, \theta}\left(s, \mathcal{B}_{\text {even }}^{(\lambda, \infty)}\right), \operatorname{Re}(s) \gg 0$ extends meromorphically to $\mathbb{C}$ and is regular at $s=0$ with the following derivative at zero:

$$
\begin{aligned}
\left.\left.\frac{d}{d s}\right|_{s=0} \zeta_{\mathrm{gr}, \theta}\left(s, \mathcal{B}_{\text {even }}^{(\lambda, \infty)}\right)\right)= & \left.\frac{1}{2} \sum_{k=0}^{m}(-1)^{k+1} \cdot k \cdot \frac{d}{d s}\right|_{s=0} \zeta_{2 \theta}\left(s, \mathcal{B}^{2} \uparrow \widetilde{\mathcal{D}}_{(\lambda, \infty)}^{k}\right) \\
& +\frac{i \pi}{2} \sum_{k=0}^{m}(-1)^{k} \cdot k \cdot \zeta_{2 \theta}\left(0, \mathcal{B}^{2} \uparrow \widetilde{\mathcal{D}}_{(\lambda, \infty)}^{k}\right)+i \pi \eta\left(\mathcal{B}_{\text {even }}^{(\lambda, \infty)}\right)
\end{aligned}
$$

Proof For $\operatorname{Re}(s) \gg 0$ the general identities [BK1 (4.10), (4.11)] imply the following relation between holomorphic functions:

$$
\begin{array}{r}
\left.\zeta_{\mathrm{gr}, \theta}\left(s, \mathcal{B}_{\text {even }}^{(\lambda, \infty)}\right)\right)=\frac{1+e^{-i \pi s}}{2}\left(\zeta_{2 \theta}\left(\frac{s}{2},\left(\mathcal{B}_{\text {even }}^{+,(\lambda, \infty)}\right)^{2}\right)-\zeta_{2 \theta}\left(\frac{s}{2},\left(\mathcal{B}_{\text {even }}^{-,(\lambda, \infty)}\right)^{2}\right)\right) \\
+\frac{1}{2}\left(1-e^{-i \pi s}\right)\left(\eta\left(s, \mathcal{B}_{\text {even }}^{(\lambda, \infty)}\right)+f(s)\right),
\end{array}
$$

where $f(s)$ is a holomorphic function (combination of zeta-functions associated to finite-dimensional operators) with

$$
f(0)=m_{+}\left(\mathcal{B}_{\text {even }}^{(\lambda, \infty)}\right)-m_{-}\left(\mathcal{B}_{\text {even }}^{(\lambda, \infty)}\right),
$$

where $m_{ \pm}(\cdot)$ denotes the number of eigenvalues of the operator in brackets, lying on the positive, respectively the negative part of the imaginary axis.

Put $\mathcal{I}=(\lambda, \infty)$ to simplify notation. Recall (3-11) and show that

$$
\widetilde{\nabla}_{\mathcal{I}}: \operatorname{ker}\left(\widetilde{\nabla}_{\mathcal{I}} \widetilde{\Gamma}_{\mathcal{I}}\right) \rightarrow \operatorname{ker}\left(\widetilde{\Gamma}_{\mathcal{I}} \widetilde{\nabla}_{\mathcal{I}}\right)=\operatorname{Im}\left(\widetilde{\nabla}_{\mathcal{I}} \widetilde{\Gamma}_{\mathcal{I}}\right)
$$

is bijective. Indeed, injectivity is clear by (3-9). For surjectivity let $x=\widetilde{\nabla}_{\mathcal{I}} \widetilde{\Gamma}_{\mathcal{I}} v \in$ $\operatorname{Im}\left(\widetilde{\nabla}_{\mathcal{I}} \widetilde{\Gamma}_{\mathcal{I}}\right)$ with (recall (3-12))

$$
v=v^{\prime} \oplus v^{\prime \prime} \in \operatorname{Im}\left(\widetilde{\nabla}_{\mathcal{I}} \widetilde{\Gamma}_{\mathcal{I}}\right) \oplus \operatorname{Im}\left(\widetilde{\Gamma}_{\mathcal{I}} \tilde{\nabla}_{\mathcal{I}}\right)=\operatorname{Im}\left(1-\Pi_{\mathcal{B}^{2},[0, \lambda]}\right) .
$$


In particular $v^{\prime \prime} \in \operatorname{Im}\left(\widetilde{\Gamma}_{\mathcal{I}} \widetilde{\nabla}_{\mathcal{I}}\right)=\operatorname{ker} \widetilde{\nabla}_{\mathcal{I}} \widetilde{\Gamma}_{\mathcal{I}}$ and $v^{\prime}=\tilde{\nabla}_{\mathcal{I}} \widetilde{\Gamma}_{\mathcal{I}} \omega$ for some $\omega$. Hence we obtain

$$
x=\widetilde{\nabla}_{\mathcal{I}} \widetilde{\Gamma}_{\mathcal{I}} v=\widetilde{\nabla}_{\mathcal{I}} \widetilde{\Gamma}_{\mathcal{I}} v^{\prime}=\widetilde{\nabla}_{\mathcal{I}} \widetilde{\Gamma}_{\mathcal{I}} \widetilde{\nabla}_{\mathcal{I}} \widetilde{\Gamma}_{\mathcal{I}} \omega
$$

and

$$
\widetilde{\Gamma}_{\mathcal{I}} \tilde{\nabla}_{\mathcal{I}} \widetilde{\Gamma}_{\mathcal{I}} \omega \in \operatorname{ker} \widetilde{\nabla}_{\mathcal{I}} \widetilde{\Gamma}_{\mathcal{I}}
$$

In other words we have found a preimage of any $x \in \operatorname{Im}\left(\widetilde{\nabla}_{\mathcal{I}} \widetilde{\Gamma}_{\mathcal{I}}\right)$ under $\tilde{\nabla}_{\mathcal{I}}$. This proves bijectivity of the map in (4-2) and consequently, since $\widetilde{\nabla}_{\mathcal{I}}$ commutes with $\mathcal{B}^{\mathcal{I}}$ and $\left(\mathcal{B}^{\mathcal{I}}\right)^{2}$, we obtain in any degree $k=0, \ldots, m$

$$
\zeta_{2 \theta}\left(s,\left(\mathcal{B}^{+, \mathcal{I}}\right)^{2} \uparrow \widetilde{\mathcal{D}}^{k}\right)=\zeta_{2 \theta}\left(s,\left(\mathcal{B}^{-, \mathcal{I}}\right)^{2} \uparrow \widetilde{\mathcal{D}}^{k+1}\right) .
$$

Using this relation we compute straightforwardly for $\operatorname{Re}(s)$ sufficiently large:

(4-4) $\zeta_{2 \theta}\left(s,\left(\mathcal{B}_{\text {even }}^{+, \mathcal{I}}\right)^{2}\right)-\zeta_{2 \theta}\left(s,\left(\mathcal{B}_{\text {even }}^{-, \mathcal{I}}\right)^{2}\right)=\sum_{k=0}^{m}(-1)^{k+1} \cdot k \cdot \zeta_{2 \theta}\left(s,\left(\mathcal{B}^{\mathcal{I}}\right)^{2} \uparrow \tilde{\mathcal{D}}^{k}\right)$

We arrive at the following preliminary result for $\operatorname{Re}(s) \gg 0$

$$
\begin{aligned}
\left.\zeta_{\mathrm{gr}, \theta}\left(s, \mathcal{B}_{\mathrm{even}}^{\mathcal{I}}\right)\right)=\frac{1}{2}\left(1+e^{-i \pi s}\right) \sum_{k=0}^{m}(-1)^{k+1} \cdot k \cdot \zeta_{2 \theta}\left(s,\left(\mathcal{B}^{\mathcal{I}}\right)^{2} \uparrow \tilde{\mathcal{D}}^{k}\right) \\
+\frac{1}{2}\left(1-e^{-i \pi s}\right)\left(\eta\left(s, \mathcal{B}_{\text {even }}^{\mathcal{I}}\right)+f(s)\right) .
\end{aligned}
$$

We find with Theorem 3.9 and Proposition 4.3 that the right hand side of the equality above is a meromorphic function on the entire complex plane and is regular at $s=0$. Hence the left hand side of the equality, the graded zeta-function, is meromorphic on $\mathbb{C}$ and regular at $s=0$, as claimed and as anticipated in Definition 3.17. Computing the derivative at zero, we obtain the statement of the theorem.

Fix now an Agmon angle $\theta \in(-\pi / 2,0)$ for $\mathcal{B}_{\text {even }}$ as in Theorem 4.4 for the remaining part of the section. As a consequence of the theorem above, we obtain for the element $\rho\left(\nabla, g^{M}\right)$ defined in Proposition 3.18 the following relation:

$$
\begin{aligned}
\rho\left(\nabla, g^{M}\right) & =e^{\xi_{\lambda}\left(\nabla, g^{M}\right)} e^{-i \pi \hat{\xi}_{\lambda}\left(\nabla, g^{M}\right)} e^{-i \pi \eta\left(\mathcal{B}_{\text {even }}^{(\lambda, \infty)}\left(g^{M}\right)\right)} \cdot \rho_{[0, \lambda]}, \\
\xi_{\lambda}\left(\nabla, g^{M}\right) & =\left.\frac{1}{2} \sum_{k=0}^{m}(-1)^{k} \cdot k \cdot \frac{d}{d s}\right|_{s=0} \zeta_{2 \theta}\left(s,\left(\mathcal{B}^{2} \uparrow \widetilde{\mathcal{D}}_{(\lambda, \infty)}^{k}\right)\right) \\
\hat{\xi}_{\lambda}\left(\nabla, g^{M}\right) & =\frac{1}{2} \sum_{k=0}^{m}(-1)^{k} \cdot k \cdot \zeta_{2 \theta}\left(s=0,\left(\mathcal{B}^{2} \uparrow \tilde{\mathcal{D}}_{(\lambda, \infty)}^{k}\right)\right) .
\end{aligned}
$$


Now we can identify explicitly the metric dependence of $\rho\left(\nabla, g^{M}\right)$ using the formula (4-6).

Consider a smooth family $g^{M}(t), t \in \mathbb{R}$ of Riemannian metrics on $M$. Denote by $\widetilde{\Gamma}_{t}$ the corresponding chirality operator in the sense of Equation (3-2) and denote the associated refined torsion (cf (3-8)) of the complex $\left(\widetilde{\mathcal{D}}_{t,[0, \lambda]}, \widetilde{\nabla}_{t,[0, \lambda]}\right)$ by $\rho_{t,[0, \lambda]}$.

Let $\mathcal{B}(t)=\mathcal{B}\left(\nabla, g^{M}(t)\right)$ be the odd-signature operator corresponding to the Riemannian metric $g^{M}(t)$. Fix $t_{0} \in \mathbb{R}$ and choose $\lambda \geq 0$ such that there are no eigenvalues of $\mathcal{B}\left(t_{0}\right)^{2}$ of absolute value $\lambda$. Then there exists $\delta>0$ small enough such that the same holds for the spectrum of $\mathcal{B}(t)^{2}$ for $\left|t-t_{0}\right|<\delta$. Under this setup we obtain:

Proposition 4.5 Let the family $g^{M}(t)$ vary only in a compact subset of the interior of $M$. Then $\exp \left(\xi_{\lambda}\left(\nabla, g^{M}(t)\right)\right) \cdot \rho_{t,[0, \lambda]}$ is independent of $t \in\left(t_{0}-\delta, t_{0}+\delta\right)$.

Proof The arguments of [6, Lemma 9.2] are of local nature and transfer ad verbatim to the present situation for metric variations in the interior of the manifold. Hence the assertion follows for Riemannian metric remaining fixed in an open neighborhood of the boundary.

Proposition 4.6 Denote the trivial connection on the trivial line bundle $M \times \mathbb{C}$ by $\nabla_{\text {trivial }}$. Consider the even part of the associated odd-signature operator (recall Definition 3.7) $\mathcal{B}_{\text {trivial }}:=\mathcal{B}_{\text {even }}\left(\nabla_{\text {trivial }}\right)$ and put

$$
\eta_{\text {trivial }}:=\frac{1}{2} \eta\left(0, \mathcal{B}_{\text {trivial }}\right)
$$

Indicate the metric dependence as follows:

$$
\begin{aligned}
\mathcal{B}_{\text {trivial }}(t) & :=\mathcal{B}_{\text {trivial }}\left(g^{M}\right), \\
\eta_{\text {trivial }}(t) & :=\frac{1}{2} \eta\left(0, \mathcal{B}_{\text {trivial }}(t)\right) .
\end{aligned}
$$

Then $\eta\left(\mathcal{B}_{\text {even }}^{(\lambda, \infty)}(t)\right)-\operatorname{rank}(E) \eta_{\text {trivial }}(t)$ is modulo $\mathbb{Z}$ independent of $t \in\left(t_{0}-\delta, t_{0}+\delta\right)$.

Proof Indicate the dependence of $\widetilde{\mathcal{D}}_{[0, \lambda]}^{*}$ on $g^{M}(t)$ by

$$
\widetilde{\mathcal{D}}_{[0, \lambda]}^{k}(t):=\text { Image } \Pi_{\mathcal{B}(t)^{2},[0, \lambda]} \cap \widetilde{\mathcal{D}}^{k} .
$$

Note first the by the choice of $\delta>0$

$$
\operatorname{dim} \widetilde{\mathcal{D}}_{[0, \lambda]}^{k}(t)=\text { const }, \quad t \in\left(t_{0}-\delta, t_{0}+\delta\right) .
$$


Since $\mathcal{B}_{\text {even }}^{[0, \lambda]}(t)$ is finite-dimensional, we infer from the definition of the eta-invariant $(\mathrm{cf}[6,(9.11)])$

$$
\eta\left(\mathcal{B}_{\text {even }}^{[0, \lambda]}(t)\right) \equiv \frac{1}{2} \operatorname{dim} \widetilde{\mathcal{D}}_{[0, \lambda]}^{\text {even }}(t) \equiv \text { const } \bmod \mathbb{Z}, \quad t \in\left(t_{0}-\delta, t_{0}+\delta\right) .
$$

By construction

$$
\eta\left(\mathcal{B}_{\text {even }}(t)\right)=\eta\left(\mathcal{B}_{\text {even }}^{(\lambda, \infty)}(t)\right)+\eta\left(\mathcal{B}_{\text {even }}^{[0, \lambda]}(t)\right) .
$$

Hence, in view of (4-9), it suffices (modulo $\mathbb{Z}$ ) to study the metric dependence of the eta-invariant of $\eta\left(\mathcal{B}_{\text {even }}(t)\right)$.

View $\mathcal{B}_{\text {even }}(t)$ as a pair of a differential operator $P_{E}(t)$ with its boundary conditions $Q_{E}(t)$. Similarly view $\mathcal{B}_{\text {trivial }}(t)$ as a pair $\left(P_{\mathbb{C}}(t), Q_{\mathbb{C}}(t)\right)$. Note that by construction the pair $\left(P_{E}(t), Q_{E}(t)\right)$ is locally isomorphic to $\left(P_{\mathbb{C}}(t), Q_{\mathbb{C}}(t)\right) \times \mathbf{1}^{k}$, since the flat connection $\nabla$ is locally trivial in appropriate local trivializations.

Since the variation of the eta-invariants is computed from the local information of the symbols (cf [18, Theorem 2.8, Lemma 2.9]), we find that the difference

$$
\eta\left(\mathcal{B}_{\text {even }}(t)\right)-\operatorname{rank}(E) \eta\left(\mathcal{B}_{\text {trivial }}(t)\right)=\eta\left(P_{E}(t), Q_{E}(t)\right)-\operatorname{rank}(E) \eta\left(P_{\mathbb{C}}(t), Q_{\mathbb{C}}(t)\right)
$$

is independent of $t \in \mathbb{R}$ modulo $\mathbb{Z}$. The modulo $\mathbb{Z}$ reduction is needed to annihilate discontinuity jumps arising from eigenvalues crossing the imaginary axis. Note that by definition

$$
\eta_{\text {trivial }}(t)=\eta\left(\mathcal{B}_{\text {trivial }}(t)\right)-\frac{1}{2} \operatorname{dim} \operatorname{ker} \mathcal{B}_{\text {trivial }}(t) .
$$

However $\operatorname{ker} \mathcal{B}_{\text {trivial }}(t) \cong H^{\text {even }}(M, \partial M, \mathbb{C}) \oplus H^{\text {even }}(M, \mathbb{C})$, the direct sum of relative and absolute cohomology of $M$ with complex coefficients in even degrees. Hence $\operatorname{dim} \operatorname{ker} \mathcal{B}_{\text {trivial }}(t)$ is in fact independent of $t \in \mathbb{R}$, which proves the statement of the proposition.

Proposition 4.7 Let $\mathcal{B}\left(\nabla_{\text {trivial }}\right)$ denote the odd-signature operator (Definition 3.7) associated to the trivial line bundle $M \times \mathbb{C}$ with the trivial connection $\nabla_{\text {trivial }}$. Consider in correspondence to (4-8) the expression

$$
\widehat{\xi}\left(\nabla_{\text {trivial }}, g^{M}(t)\right)=\frac{1}{2} \sum_{k=0}^{m}(-1)^{k} \cdot k \cdot \zeta_{2 \theta}\left(s=0,\left(\mathcal{B}\left(\nabla_{\text {trivial }}, g^{M}(t)\right)^{2} \uparrow \widetilde{\mathcal{D}}^{k}\right) .\right.
$$

Then

$$
\widehat{\xi}_{\lambda}\left(\nabla, g^{M}(t)\right)-\operatorname{rank}(E) \cdot \hat{\xi}\left(\nabla_{\text {trivial }}, g^{M}(t)\right) \bmod \mathbb{Z}
$$

is independent of $t \in \mathbb{R}$. 
Proof We show first that modulo $\mathbb{Z}$ it suffices to study the metric dependence of

$$
\widehat{\xi}\left(\nabla, g^{M}(t)\right):=\frac{1}{2} \sum_{k=0}^{m}(-1)^{k} \cdot k \cdot \zeta_{2 \theta}\left(s=0,\left(\mathcal{B}\left(\nabla, g^{M}(t)\right)^{2} \uparrow \widetilde{\mathcal{D}}^{k}\right) .\right.
$$

Indeed, by construction we have

$$
\widehat{\xi}\left(\nabla, g^{M}(t)\right)=\widehat{\xi}_{\lambda}\left(\nabla, g^{M}(t)\right)+\frac{1}{2} \sum_{k=0}^{m}(-1)^{k} \cdot k \cdot \operatorname{dim} \widetilde{\mathcal{D}}_{(0, \lambda]}^{k}(t) .
$$

Anticipating the auxiliary result of Lemma 4.8 (iii) below, we obtain

$$
\widehat{\xi}\left(\nabla, g^{M}(t)\right) \equiv \widehat{\xi}_{\lambda}\left(\nabla, g^{M}(t)\right) \bmod \mathbb{Z} .
$$

Recall that $\mathcal{B}\left(\nabla_{\text {trivial }}, g^{M}\right) \times \mathbf{1}^{\mathrm{rk} E}$ and $\mathcal{B}\left(\nabla, g^{M}\right)$ are locally isomorphic, as already encountered in the proof of Proposition 4.6. Now the statement of the proposition follows from the fact that the value of a zeta function at zero is given, modulo $\mathbb{Z}$ in order to avoid $\operatorname{dim} \operatorname{ker} \mathcal{B}(t) \in \mathbb{Z}$, by integrands of local invariants of the operator and its boundary conditions.

Lemma 4.8 Let $\mathcal{I} \subset \mathbb{R}$ denote any bounded interval. Then:

(i) $\frac{1}{2} \sum_{k=0}^{m}(-1)^{k+1} \cdot k \cdot \operatorname{dim} \widetilde{\mathcal{D}}_{\mathcal{I}}^{k} \equiv(\operatorname{dim} M / 2) \operatorname{dim} \widetilde{\mathcal{D}}_{\mathcal{I}}^{\text {even }} \bmod 2 \mathbb{Z}$.

(ii) If $0 \notin \mathcal{I}$, then $\operatorname{dim} \widetilde{\mathcal{D}}_{\mathcal{I}}^{\text {even }} \equiv 0 \bmod 2 \mathbb{Z}$,

(iii) If $0 \notin \mathcal{I}$, then $\frac{1}{2} \sum_{k=0}^{m}(-1)^{k+1} \cdot k \cdot \operatorname{dim} \widetilde{\mathcal{D}}_{\mathcal{I}}^{k} \equiv 0 \bmod \mathbb{Z}$.

Proof Note first the following relation:

$$
\mathcal{B}_{k}^{2}=\widetilde{\Gamma} \circ \mathcal{B}_{m-k}^{2} \circ \widetilde{\Gamma}
$$

Hence with $r=(m+1) / 2$ we obtain

$$
\begin{aligned}
\frac{1}{2} \sum_{k=0}^{m}(-1)^{k+1} \cdot k \cdot \operatorname{dim} \widetilde{\mathcal{D}}_{\mathcal{I}}^{k} & =\frac{1}{2} \sum_{k=0}^{r-1}(m-4 k) \cdot \operatorname{dim} \widetilde{\mathcal{D}}_{\mathcal{I}}^{2 k} \\
& =\frac{m}{2} \operatorname{dim} \widetilde{\mathcal{D}}_{\mathcal{I}}^{\text {even }}-2 \sum_{k=0}^{r-1} k \cdot \operatorname{dim} \widetilde{\mathcal{D}}_{\mathcal{I}}^{2 k} .
\end{aligned}
$$

This proves the first statement. For the second statement assume $0 \notin \mathcal{I}$ till the end of the proof. Consider the operators

$$
\begin{aligned}
& \mathcal{B}_{k}^{+, \mathcal{I}}=\widetilde{\Gamma}_{\mathcal{I}} \widetilde{\nabla}_{\mathcal{I}}: \widetilde{\mathcal{D}}_{\mathcal{I}}^{k} \cap \operatorname{ker}\left(\widetilde{\nabla}_{\mathcal{I}} \widetilde{\Gamma}_{\mathcal{I}}\right) \rightarrow \widetilde{\mathcal{D}}_{\mathcal{I}}^{m-k-1} \cap \operatorname{ker}\left(\widetilde{\nabla}_{\mathcal{I}} \widetilde{\Gamma}_{\mathcal{I}}\right), \\
& \mathcal{B}_{k}^{-, \mathcal{I}}=\widetilde{\nabla}_{\mathcal{I}} \widetilde{\Gamma}_{\mathcal{I}}: \widetilde{\mathcal{D}}_{\mathcal{I}}^{k} \cap \operatorname{ker}\left(\widetilde{\Gamma}_{\mathcal{I}} \tilde{\nabla}_{\mathcal{I}}\right) \rightarrow \widetilde{\mathcal{D}}_{\mathcal{I}}^{m-k+1} \cap \operatorname{ker}\left(\widetilde{\Gamma}_{\mathcal{I}} \widetilde{\nabla}_{\mathcal{I}}\right) .
\end{aligned}
$$


Since $0 \notin \mathcal{I}$, the maps $\mathcal{B}_{k}^{ \pm, \mathcal{I}}$ are isomorphisms by bijectivity of the map in (4-2). Furthermore they commute with $\left(\mathcal{B}^{ \pm, \mathcal{I}}\right)^{2}$ in the following way:

$$
\mathcal{B}_{k}^{ \pm, \mathcal{I}} \circ\left[\left(\mathcal{B}^{ \pm, \mathcal{I}}\right)^{2} \uparrow \widetilde{\mathcal{D}}^{k}\right]=\left[\left(\mathcal{B}^{ \pm, \mathcal{I}}\right)^{2} \uparrow \widetilde{\mathcal{D}}^{m-k \mp 1}\right] \circ \mathcal{B}_{k}^{ \pm, \mathcal{I}}
$$

Hence we obtain, denoting by $\widetilde{\mathcal{D}}_{\mathcal{I}}^{ \pm, k}$ the span of generalized eigenforms of $\left(\mathcal{B}^{ \pm, \mathcal{I}}\right)^{2} \uparrow \widetilde{\mathcal{D}}^{k}$, the relations

$$
\begin{aligned}
\operatorname{dim} \widetilde{\mathcal{D}}_{\mathcal{I}}^{+, k} & =\operatorname{dim} \widetilde{\mathcal{D}}_{\mathcal{I}}^{+, m-k-1}, \\
\operatorname{dim} \widetilde{\mathcal{D}}_{\mathcal{I}}^{-, k} & =\operatorname{dim} \widetilde{\mathcal{D}}_{\mathcal{I}}^{-, m-k+1} .
\end{aligned}
$$

As $\operatorname{dim} \widetilde{\mathcal{D}}_{\mathcal{I}}^{\text {even }}=\operatorname{dim} \widetilde{\mathcal{D}}_{\mathcal{I}}^{+ \text {,even }}+\operatorname{dim} \widetilde{\mathcal{D}}_{\mathcal{I}}^{- \text {,even }}$, this implies (recall $M$ is odd-dimensional)

$$
\begin{aligned}
& \operatorname{dim} \widetilde{\mathcal{D}}_{\mathcal{I}}^{\text {even }} \equiv \operatorname{dim} \widetilde{\mathcal{D}}_{\mathcal{I}}^{+, 2 p} \bmod 2 \mathbb{Z}, \text { if } \operatorname{dim} M=4 p+1, \\
& \operatorname{dim} \widetilde{\mathcal{D}}_{\mathcal{I}}^{\text {even }} \equiv \operatorname{dim} \widetilde{\mathcal{D}}_{\mathcal{I}}^{-, 2 p} \bmod 2 \mathbb{Z}, \text { if } \operatorname{dim} M=4 p-1 .
\end{aligned}
$$

Finally recall the explicit form of $\left(\mathcal{B}^{ \pm}\right)^{2}$ :

$$
\begin{aligned}
\left(\mathcal{B}^{+}\right)^{2} & =\left(\begin{array}{cc}
\Gamma \nabla_{\max } \Gamma \nabla_{\min } & 0 \\
0 & \Gamma \nabla_{\min } \Gamma \nabla_{\max }
\end{array}\right)=:\left(\begin{array}{cc}
D_{1}^{+} & 0 \\
0 & D_{2}^{+}
\end{array}\right), \\
\left(\mathcal{B}^{-}\right)^{2} & =\left(\begin{array}{cc}
\nabla_{\min } \Gamma \nabla_{\max } \Gamma & 0 \\
0 & \nabla_{\max } \Gamma \nabla_{\min } \Gamma
\end{array}\right)=:\left(\begin{array}{cc}
D_{1}^{-} & 0 \\
0 & D_{2}^{-}
\end{array}\right) .
\end{aligned}
$$

Moreover we put

$$
\left(\mathcal{B}^{ \pm, \mathcal{I}}\right)^{2} \uparrow \widetilde{\mathcal{D}}^{k}=D_{1, k}^{ \pm, \mathcal{I}} \oplus D_{2, k}^{ \pm, \mathcal{I}}
$$

Note the relations

$$
\begin{aligned}
& \left(\Gamma \nabla_{\min }\right) \circ D_{1}^{+}=D_{2}^{+} \circ\left(\Gamma \nabla_{\min }\right), \\
& D_{1}^{+} \circ\left(\Gamma \nabla_{\max }\right)=\left(\Gamma \nabla_{\max }\right) \circ D_{2}^{+} ; \\
& \left(\nabla_{\max } \Gamma\right) \circ D_{1}^{-}=D_{2}^{-} \circ\left(\nabla_{\max } \Gamma\right), \\
& D_{1}^{-} \circ\left(\nabla_{\min } \Gamma\right)=\left(\nabla_{\min } \Gamma\right) \circ D_{2}^{-} .
\end{aligned}
$$

Due to $0 \notin \mathcal{I}$ these relations imply, similarly to (4-13), spectral equivalence of $D_{1, k}^{ \pm, \mathcal{I}}$ and $D_{2, k}^{ \pm, \mathcal{I}}$ in the middle degree $k=2 p$ for $\operatorname{dim} M=4 p \pm 1$, respectively. This finally yields the desired relations:

$\operatorname{dim} \widetilde{\mathcal{D}}_{\mathcal{I}}^{\text {even }} \equiv \operatorname{dim} \widetilde{\mathcal{D}}_{\mathcal{I}}^{+, 2 p} \equiv 0 \bmod 2 \mathbb{Z}, \quad$ if $\operatorname{dim} M=4 p+1$, $\operatorname{dim} \widetilde{\mathcal{D}}_{\mathcal{I}}^{\text {even }} \equiv \operatorname{dim} \widetilde{\mathcal{D}}_{\mathcal{I}}^{-, 2 p} \equiv 0 \bmod 2 \mathbb{Z}, \quad$ if $\operatorname{dim} M=4 p-1$. 
Propositions 4.5, 4.6 and 4.7 determine together the metric anomaly of $\rho\left(\nabla, g^{M}\right)$ up to a sign and we deduce the following central corollary.

Corollary 4.9 Let $M$ be an odd-dimensional oriented compact Riemannian manifold. Let $\left(E, \nabla, h^{E}\right)$ be a flat complex vector bundle over $M$. Denote by $\nabla_{\text {trivial }}$ the trivial connection on $M \times \mathbb{C}$ and let $\mathcal{B}_{\text {trivial }}$ denote the even part of the associated odd-signature operator. Then

$$
\rho_{\text {an }}(\nabla):=\rho\left(\nabla, g^{M}\right) \cdot \exp \left(i \pi \operatorname{rk}(E)\left(\eta_{\text {trivial }}\left(g^{M}\right)+\hat{\xi}\left(\nabla_{\text {trivial }}, g^{M}\right)\right)\right)
$$

is independent of the choice of $g^{M}$ in the interior of $M$.

Proof Consider a smooth family $g^{M}(t), t \in \mathbb{R}$ of Riemannian metrics, varying only in the interior of $M$. Then $\rho\left(\nabla, g^{M}(t)\right)$ is continuous in $t$, due to well-known continuity of zeta-determinants; compare [11, Section 3.7]. Similarly we have continuity of $\hat{\xi}\left(\nabla_{\text {trivial }}, g^{M}(t)\right)$. The additional term

$$
\exp \left(i \pi \operatorname{rk}(E) \eta_{\text {trivial }}\left(g^{M}(t)\right)\right)
$$

is also continuous in $t \in \mathbb{R}$. For this note that the kernel of $\mathcal{B}_{\text {trivial }}$ is given up to isomorphism by a direct sum of relative and absolute cohomology of $M$ with complex coefficients in even degrees

$$
\operatorname{ker} \mathcal{B}_{\text {trivial }}\left(g^{M}(t)\right) \cong H^{\text {even }}(M, \partial M, \mathbb{C}) \oplus H^{\text {even }}(M, \mathbb{C})
$$

Hence $\operatorname{dim} \operatorname{ker} \mathcal{B}_{\text {trivial }}\left(g^{M}(t)\right)$ is in fact independent of $t \in \mathbb{R}$ and thus no eigenvalues of $\mathcal{B}_{\text {trivial }}\left(g^{M}(t)\right)$ can cross zero, as $t$ varies. Therefore $\rho_{\text {an }}(\nabla)$, where we denote its a priori metric dependence by $\rho_{\mathrm{an}}\left(\nabla, g^{M}(t)\right)$, is continuous and in $t$ and nowhere vanishing.

For $g^{M}(t)$ varying only in the interior of $M$ and any $t_{0}, t_{1} \in \mathbb{R}$ we infer from the $\bmod \mathbb{Z}$ metric anomaly considerations in Proposition 4.6 and Proposition 4.7:

$$
\rho_{\mathrm{an}}\left(\nabla, g^{M}\left(t_{0}\right)\right)= \pm \rho_{\mathrm{an}}\left(\nabla, g^{M}\left(t_{1}\right)\right) .
$$

Since $\rho_{\mathrm{an}}\left(\nabla, g^{M}(t)\right)$ is continuous and nowhere vanishing, the sign at the right hand side of the equality must be positive. This proves the statement.

In view of the corollary above we can now define the "refined analytic torsion". It will be a differential invariant in the sense, that even though defined by geometric data in form of the metric structures, it is shown to be independent of their form in the interior of the manifold. 
Definition 4.10 Let $M$ be an odd-dimensional oriented Riemannian manifold. Let $(E, \nabla)$ be a flat complex vector bundle over $M$. The refined analytic torsion $\rho_{\text {an }}(\nabla)$ is an element of $\operatorname{Det} H^{*}(\widetilde{\mathcal{D}}, \widetilde{\nabla})$, defined by (4-16).

\section{Comparison with Braverman and Kappeler's refined ana- lytic torsion}

Let $\left(M, g^{M}\right)$ be a compact odd-dimensional oriented Riemannian manifold with boundary $\partial M$, which may be empty. Let $\left(E, \nabla, h^{E}\right)$ be a flat complex vector bundle over $M$. The Hermitian metric $h^{E}$ need not to be flat with respect to the connection $\nabla$. The refined analytic torsion by Braverman and Kappeler in [6, Definition 9.8] refines analytic Ray-Singer torsion on closed manifolds. It can be applied to the Riemannian manifold $\left(M, g^{M}\right)$ with the flat complex vector bundle $(E, \nabla)$ in case the boundary $\partial M$ is empty.

Assume $\partial M=\varnothing$ and denote the associated odd signature operator, in the sense of [6, Definition 7.3] by $B$ and its even part by $B_{\text {even. The even part of the odd signature }}$ operator in the sense of [6, Definition 7.3], associated to the trivial line bundle $M \times \mathbb{C}$, shall be denoted by $B_{\text {trivial }}$. For any $\lambda \geq 0$ one can decompose

$$
B_{\text {even }}=B_{\text {even }}^{[0, \lambda]} \oplus B_{\text {even }}^{(\lambda, \infty)},
$$

similar to (3-7) and further define, following [6, Section 1.1] the associated refined torsion element $\rho_{[0, \lambda]}^{\mathrm{BK}} \in \operatorname{det} H^{*}(M, E)$. Then the refined analytic torsion of Braverman and Kappeler is given by

$$
\rho_{\mathrm{an}}^{\mathrm{BK}}(\nabla)=\operatorname{det}_{\mathrm{gr}}\left(B_{\text {even }}^{(\lambda, \infty)}\right) \cdot \exp \left(i \pi \mathrm{rk}(E) \cdot \frac{1}{2} \eta\left(0, B_{\text {trivial }}\right)\right) \cdot \rho_{[0, \lambda]}^{\mathrm{BK}} .
$$

The construction of Braverman and Kappeler does not apply to manifolds with boundary. Our construction of refined analytic torsion closes this gap, seems however slightly more complicated. To illustrate the differences more precisely, let us apply our construction to $\left(M, g^{M}\right)$ in case of nonempty boundary $\partial M$.

Denote the associated odd signature operator in the sense of Definition 3.7 by $\mathcal{B}$ and its even part by $\mathcal{B}_{\text {even. }}$. The even part of the odd signature operator in the sense of Definition 3.7, associated to the trivial line bundle $M \times \mathbb{C}$, shall be denoted by $\mathcal{B}_{\text {trivial }}$. Moreover we consider the expression $\hat{\xi}\left(\nabla_{\text {trivial }}, g^{M}\right)$, defined in Proposition 4.7 in terms of zeta-functions associated to $\mathcal{B}_{\text {trivial }}$.

For any $\lambda \geq 0$ we can decompose

$$
\mathcal{B}_{\text {even }}=\mathcal{B}_{\text {even }}^{[0, \lambda]} \oplus \mathcal{B}_{\text {even }}^{(\lambda, \infty)}
$$


as in (3-7) and further consider, following (3-8), the associated refined torsion element $\rho_{[0, \lambda]}$. Then our definition of refined analytic torsion in Definition 4.10 is given by

$$
\rho_{\mathrm{an}}(\nabla)=\operatorname{det}_{\mathrm{gr}}\left(\mathcal{B}_{\text {even }}^{(\lambda, \infty)}\right) \cdot \exp \left(i \pi \operatorname{rk}(E)\left(\frac{1}{2} \eta\left(0, \mathcal{B}_{\text {trivial }}\right)+\hat{\xi}\left(\nabla_{\text {trivial }}, g^{M}\right)\right)\right) \cdot \rho_{[0, \lambda]} .
$$

Formal comparison of expressions $\rho_{\mathrm{an}}^{\mathrm{BK}}(\nabla)$ and $\rho_{\mathrm{an}}(\nabla)$ shows that in contrast to refined analytic torsion of Braverman and Kappeler, the complex phase of our construction contains the term $\hat{\xi}\left(\nabla_{\text {trivial }}, g^{M}\right)$ in addition to the eta-invariant term.

Below we discuss the origin of the additional term $\hat{\xi}\left(\nabla_{\text {trivial }}, g^{M}\right)$ in our construction of refined analytic torsion. Furthermore we compare both constructions on a closed Riemannian manifold.

\subsection{Origin of the additional term $\hat{\xi}$ in the complex phase}

The additional term $\hat{\xi}\left(\nabla_{\text {trivial }}, g^{M}\right)$ is designed to annihilate the metric dependence of $\hat{\xi}_{\lambda}\left(\nabla, g^{M}\right), \lambda \geq 0$. The term $\hat{\xi}_{\lambda}\left(\nabla, g^{M}\right)$ appears in Theorem 4.4 and is a weighted alternating sum of zeta functions of $\mathcal{B}^{2} \uparrow \widetilde{\mathcal{D}}_{(\lambda, \infty)}^{k}$, evaluated at zero. Similar term appears in the construction of refined analytic torsion in [6], as well.

However, on closed odd-dimensional manifolds zeta-functions $\zeta_{\theta}(s, D)$ of bijective differential operators of even order vanish at $s=0$ and the general result [6, Proposition 6.5] holds. As a consequence, in case of empty boundary there is no metric dependence to annihilate, other than that coming from the eta-invariant.

On compact odd-dimensional manifolds with boundary, zeta-functions of bijective elliptic operators of even order need not to vanish at zero and hence we cannot apply the general arguments of [6]. In particular the metric dependence of $\hat{\xi}_{\lambda}\left(\nabla, g^{M}\right)$ may be nontrivial.

If however $\partial M=\varnothing$, then by the arguments of [6, Proposition 6.5] and Lemma 4.8 we obtain for the additional term $\hat{\xi}\left(\nabla_{\text {trivial }}, g^{M}\right)$

$$
\widehat{\xi}\left(\nabla_{\text {trivial }}, g^{M}\right)=\frac{\operatorname{dim} M}{2} \cdot \operatorname{dim} H^{\text {even }}(M, \mathbb{C})
$$

Hence $\hat{\xi}\left(\nabla_{\text {trivial }}, g^{M}\right)$ does not depend on the Riemannian metric $g^{M}$ in case of empty boundary. In particular there is no metric dependence of $\hat{\xi}_{\lambda}\left(\nabla, g^{M}\right)$ to annihilate and in the setup of closed Riemannian manifolds indeed only an additional complex phase $\exp \left(i \pi \operatorname{rk}(E) \cdot \frac{1}{2} \eta\left(0, \mathcal{B}_{\text {trivial }}\right)\right)$ is required to turn $\operatorname{det}_{\mathrm{gr}}\left(\mathcal{B}_{\text {even }}^{(\lambda, \infty)}\right) \cdot \rho_{[0, \lambda]}$ into a differential invariant. 
Similar situation appears on compact manifolds with product metric structures near the boundary, as in the setup of Theorem 6.3 below. In this case one can relate the constructions to a closed smooth double manifold and argue as in the boundaryless case.

\subsection{Comparison of both constructions on a closed manifold}

Theorem 5.1 Let $\left(M, g^{M}\right)$ be a closed odd-dimensional oriented Riemannian manifold with a flat complex vector bundle $\left(E, \nabla, h^{E}\right)$ over $M$. Let

$$
\mu: \operatorname{det} H^{*}(M, E) \otimes \operatorname{det} H^{*}(M, E) \rightarrow \operatorname{det}\left(H^{*}(M, E) \oplus H^{*}(M, E)\right)
$$

be the fusion isomorphism of [6, Section 2.6]. Then we have in the notation of Section 5

$$
\mu\left(\rho_{\mathrm{an}}^{\mathrm{BK}}(\nabla) \otimes \rho_{\mathrm{an}}^{\mathrm{BK}}(\nabla)\right)=\rho_{\mathrm{an}}(\nabla) \cdot \exp (i \pi A),
$$

where

$$
\begin{aligned}
A:=-2 \eta\left(B_{\text {even }}^{(\lambda, \infty)}\right) & +\eta\left(\mathcal{B}_{\text {even }}^{(\lambda, \infty)}\right)+\operatorname{rk}(E) \eta\left(0, B_{\text {trivial }}\right) \\
& -\operatorname{rk}(E) \cdot \frac{1}{2} \eta\left(0, \mathcal{B}_{\text {trivial }}\right)-\operatorname{rk}(E) \cdot \frac{\operatorname{dim} M}{2} \cdot \operatorname{dim} H^{\text {even }}(M, \mathbb{C}) .
\end{aligned}
$$

Proof Consider the usual odd signature operator on the flat vector bundle $(E, \nabla)$ over the closed Riemannian manifold $\left(M, g^{M}\right)$

$$
B=\Gamma \nabla+\nabla \Gamma: \Omega^{*}(M, E) \rightarrow \Omega^{*}(M, E) .
$$

The odd signature operator in the sense of Definition 3.7, acting on smooth differential forms, is then given by

$$
\mathcal{B}=\left(\begin{array}{cc}
0 & B \\
B & 0
\end{array}\right): \Omega^{*}(M, E) \oplus \Omega^{*}(M, E) \rightarrow \Omega^{*}(M, E) \oplus \Omega^{*}(M, E) .
$$

Consider for any fixed $\lambda \geq 0$ the graded determinant $\operatorname{det}_{\mathrm{gr}} B_{\mathrm{even}}^{(\lambda, \infty)}$, defined in [6, (7-10)], and the graded determinant $\operatorname{det}_{\mathrm{gr}} \mathcal{B}_{\text {even }}^{(\lambda, \infty)}$ of Definition 3.17 .

Note that $\operatorname{Spec}\left(B^{2}\right)$ and $\operatorname{Spec}\left(\mathcal{B}^{2}\right)$ differ only by their multiplicities. Namely, the multiplicity of any fixed eigenvalue in $\operatorname{Spec}\left(\mathcal{B}^{2}\right)$ is double the multiplicity of this eigenvalue in $\operatorname{Spec}\left(B^{2}\right)$. Hence we find, using the relations [6, Proposition 8.1] and Theorem 4.4, for the graded determinants

$$
\operatorname{det}_{\mathrm{gr}} \mathcal{B}_{\text {even }}^{(\lambda, \infty)}=\left(\operatorname{det}_{\mathrm{gr}} B_{\text {even }}^{(\lambda, \infty)}\right)^{2} \cdot \exp \left(2 i \pi \eta\left(B_{\text {even }}^{(\lambda, \infty)}\right)-i \pi \eta\left(\mathcal{B}_{\text {even }}^{(\lambda, \infty)}\right)\right) .
$$


Furthermore, let us consider for the fixed $\lambda \geq 0$ the refined torsion elements $\rho_{[0, \lambda]}^{\mathrm{BK}} \in$ $\operatorname{det} H^{*}(M, E)$ and $\rho_{[0, \lambda]} \in \operatorname{det}\left(H^{*}(M, E) \oplus H^{*}(M, E)\right)$. Similar arguments as in [6, Lemma 4.7] show

$$
\mu\left(\rho_{[0, \lambda]}^{\mathrm{BK}} \otimes \rho_{[0, \lambda]}^{\mathrm{BK}}\right)=\rho_{[0, \lambda]} .
$$

Combination of (5-3) and (5-4), together with the formula (5-2) for $\hat{\xi}\left(\nabla_{\text {trivial }}, g^{M}\right)$ leads then to the desired result.

\section{Ray-Singer norm of refined analytic torsion}

Recall first the construction of the Ray-Singer torsion as a norm on the determinant line bundle for compact oriented Riemannian manifolds. Let $\left(M, g^{M}\right)$ and $\left(E, \nabla, h^{E}\right)$ be as in Section 3.

Let $\triangle_{\text {rel }}$ be the Laplacian associated to the Fredholm complex $\left(\mathcal{D}_{\min }, \nabla_{\min }\right)$ defined at the beginning of Section 3. As in (3-6) in case of the squared odd-signature operator $\mathcal{B}^{2}$, it induces a spectral decomposition into a direct sum of subcomplexes for any $\lambda \geq 0$.

$$
\left(\mathcal{D}_{\min }, \nabla_{\min }\right)=\left(\mathcal{D}_{\min }^{[0, \lambda]}, \nabla_{\min }^{[0, \lambda]}\right) \oplus\left(\mathcal{D}_{\min }^{(\lambda, \infty)}, \nabla_{\min }^{(\lambda, \infty)}\right) .
$$

The scalar product on $\mathcal{D}_{\text {min }}^{[0, \lambda]}$ induced by $g^{M}$ and $h^{E}$ induces a norm on the determinant line $\operatorname{Det}\left(\mathcal{D}_{\min }^{[0, \lambda]}, \nabla_{\min }^{[0, \lambda]}\right)$ (we use the notation of determinant lines of finite dimensional complexes in [6, Section 1.1]). There is a canonical isomorphism

$$
\phi_{\lambda}: \operatorname{Det}\left(\mathcal{D}_{\min }^{[0, \lambda]}, \nabla_{\min }^{[0, \lambda]}\right) \rightarrow \operatorname{Det} H^{*}\left(\mathcal{D}_{\min }, \nabla_{\min }\right),
$$

induced by the Hodge decomposition in finite-dimensional complexes. Choose on $\operatorname{Det} H^{*}\left(\mathcal{D}_{\min }, \nabla_{\min }\right)$ the norm $\|\cdot\|_{\lambda}^{\text {rel }}$ such that $\phi_{\lambda}$ becomes an isometry. Further denote by $T_{(\lambda, \infty)}^{\mathrm{RS}}\left(\nabla_{\min }\right)$ the scalar analytic torsion associated to the complex $\left(\mathcal{D}_{\min }^{(\lambda, \infty)}, \nabla_{\min }^{(\lambda, \infty)}\right)$ :

$$
T_{(\lambda, \infty)}^{\mathrm{RS}}\left(\nabla_{\min }\right):=\exp \left(\frac{1}{2} \sum_{k=1}^{m}(-1)^{k+1} \cdot k \cdot \zeta^{\prime}\left(s=0, \triangle_{k, \text { rel }}^{(\lambda, \infty)}\right)\right),
$$

where $\triangle_{\text {rel }}^{(\lambda, \infty)}$ is the Laplacian associated to the complex $\left(\mathcal{D}_{\min }^{(\lambda, \infty)}, \nabla_{\min }^{(\lambda, \infty)}\right)$. Note the difference to the sign convention of [26]. However we are consistent with [6].

The Ray-Singer norm on $\operatorname{Det} H^{*}\left(\mathcal{D}_{\min }, \nabla_{\min }\right)$ is then defined by

$$
\|\cdot\|_{\operatorname{Det} H^{*}\left(\mathcal{D}_{\min }, \nabla_{\min }\right)}^{\mathrm{RS}}:=\|\cdot\|_{\lambda}^{\mathrm{rel}} \cdot T_{(\lambda, \infty)}^{\mathrm{RS}}\left(\nabla_{\min }\right)
$$


With a completely analogous construction we obtain the Ray-Singer norm on the determinant line $\operatorname{Det} H^{*}\left(\mathcal{D}_{\max }, \nabla_{\max }\right)$

$$
\|\cdot\|_{\operatorname{Det} H^{*}\left(\mathcal{D}_{\max }, \nabla_{\max }\right)}^{\mathrm{RS}}:=\|\cdot\|_{\lambda}^{\mathrm{abs}} \cdot T_{(\lambda, \infty)}^{\mathrm{RS}}\left(\nabla_{\max }\right) .
$$

Both constructions turn out to be independent of the choice of $\lambda \geq 0$, which follows from arguments analogous to those in the proof of Proposition 3.18. In fact we get for $0 \leq \lambda<\mu$,

$$
\|\cdot\|_{\mu}^{\mathrm{rel} / \mathrm{abs}}=\|\cdot\|_{\lambda}^{\mathrm{rel} / \mathrm{abs}} \cdot T_{(\lambda, \mu]}^{\mathrm{RS}}\left(\nabla_{\min / \max }\right),
$$

which implies that the Ray-Singer norms are well-defined. Furthermore by the arguments in [24, Theorem 2.6] the norms do not depend on the metric structures in the interior of the manifold.

Remark 6.1 Note that the Ray-Singer analytic torsion considered in [33] and [23] differs from our setup in the sign convention and by the absence of factor $1 / 2$.

We can apply the same construction to the Laplacian of the complex $(\widetilde{\mathcal{D}}, \widetilde{\nabla})$ introduced in Definition 3.4

$$
(\tilde{\mathcal{D}}, \tilde{\nabla})=\left(\mathcal{D}_{\min }, \nabla_{\min }\right) \oplus\left(\mathcal{D}_{\max }, \nabla_{\max }\right) .
$$

Similarly we obtain

$$
\|\cdot\|_{\operatorname{Det} H^{*}(\tilde{\mathcal{D}}, \tilde{\nabla})}^{\mathrm{RS}}:=\|\cdot\|_{\lambda} \cdot T_{(\lambda, \infty)}^{\mathrm{RS}}(\widetilde{\nabla}) .
$$

This "doubled" Ray-Singer norm is naturally related to the previous two norms in (6-1) and (6-2). There is a canonical "fusion isomorphism"; see [6, (2.18)] for general complexes of finite dimensional vector spaces

$$
\mu: \operatorname{Det} H^{*}\left(\mathcal{D}_{\text {min }}, \nabla_{\text {min }}\right) \oplus \operatorname{Det} H^{*}\left(\mathcal{D}_{\text {max }}, \nabla_{\text {max }}\right) \rightarrow \operatorname{Det} H^{*}(\tilde{\mathcal{D}}, \tilde{\nabla}),
$$

such that

$$
\left\|\mu\left(h_{1} \otimes h_{2}\right)\right\|_{\lambda}=\left\|h_{1}\right\|_{\lambda}^{\mathrm{rel}} \cdot\left\|h_{2}\right\|_{\lambda}^{\mathrm{abs}}
$$

where we recall $(\tilde{\mathcal{D}}, \widetilde{\nabla})=\left(\mathcal{D}_{\min }, \nabla_{\min }\right) \oplus\left(\mathcal{D}_{\max }, \nabla_{\max }\right)$ by definition. Further we have by the definition of $(\widetilde{\mathcal{D}}, \widetilde{\nabla})$ following relation between the scalar analytic torsions:

$$
T_{(\lambda, \infty)}^{\mathrm{RS}}(\tilde{\nabla})=T_{(\lambda, \infty)}^{\mathrm{RS}}\left(\nabla_{\min }\right) \cdot T_{(\lambda, \infty)}^{\mathrm{RS}}\left(\nabla_{\max }\right) .
$$

Combining (6-4) and (6-5) we end up with a relation between norms

$$
\left\|\mu\left(h_{1} \otimes h_{2}\right)\right\|_{\operatorname{Det} H^{*}(\tilde{\mathcal{D}}, \tilde{\nabla})}^{\mathrm{RS}}=\left\|h_{1}\right\|_{\operatorname{Det} H^{*}\left(\mathcal{D}_{\min }, \nabla_{\min }\right)}^{\mathrm{RS}} \cdot\left\|h_{2}\right\|_{\operatorname{Det} H^{*}\left(\mathcal{D}_{\max }, \nabla_{\max }\right)}^{\mathrm{RS}} \cdot
$$

The next theorem provides a motivation for viewing $\rho_{\mathrm{an}}(\nabla)$ as a refinement of the Ray-Singer torsion. 
Theorem 6.2 Let $M$ be a smooth compact odd-dimensional oriented Riemannian manifold. Let $\left(E, \nabla, h^{E}\right)$ be a flat complex vector bundle over $M$ with a flat Hermitian metric $h^{E}$. Then

$$
\left\|\rho_{\mathrm{an}}(\nabla)\right\|_{\operatorname{Det} H^{*}(\widetilde{\mathcal{D}}, \tilde{\nabla})}^{\mathrm{RS}}=1
$$

Proof Recall from the assertion of Theorem 4.4

$$
\operatorname{det}_{\mathrm{gr}}\left(\mathcal{B}_{\text {even }}^{(\lambda, \infty)}\right)=e^{\xi_{\lambda}\left(\nabla, g^{M}\right)} \cdot e^{-i \pi \widehat{\xi}_{\lambda}\left(\nabla, g^{M}\right)} \cdot e^{-i \pi \eta\left(\mathcal{B}_{\text {even }}\right)},
$$

Flatness of $h^{E}$ implies by construction that $\mathcal{B}^{2}=\triangle_{\text {rel }} \oplus \triangle_{\text {abs }}$ and hence

$$
\xi_{\lambda}\left(\nabla, g^{M}\right)=-\log T_{(\lambda, \infty)}^{\mathrm{RS}}(\widetilde{\nabla})
$$

Further $\mathcal{B}_{\text {even }}$ is self-adjoint and thus has a real spectrum. Hence $\eta\left(\mathcal{B}_{\text {even }}\right)$ and $\hat{\xi}_{\lambda}\left(\nabla, g^{M}\right)$ are real-valued, as well. Thus we derive

$$
\left|\operatorname{det}_{\mathrm{gr}}\left(\mathcal{B}_{\text {even }}^{(\lambda, \infty)}\right)\right|=\frac{1}{T_{(\lambda, \infty)}^{\mathrm{RS}}(\widetilde{\nabla})} .
$$

Furthermore we know from [6, Lemma 4.5], which is a general result for complexes of finite-dimensional vector spaces,

$$
\left\|\rho_{[0, \lambda]}\right\|_{\lambda}=1 .
$$

Now the assertion follows by combining the definition of the refined analytic torsion with (6-7), (6-8) and the fact that the additional terms annihilating the metric anomaly are all of norm one. In fact we have

$$
\left\|\rho_{\mathrm{an}}(\nabla)\right\|_{\operatorname{Det} H^{*}(\tilde{\mathcal{D}}, \tilde{\nabla})}^{\mathrm{RS}}=\left|\operatorname{det}_{\mathrm{gr}}\left(\mathcal{B}_{\text {even }}^{(\lambda, \infty)}\right)\right| \cdot T_{(\lambda, \infty)}^{\mathrm{RS}}(\tilde{\nabla}) \cdot\left\|\rho_{[0, \lambda]}\right\|_{\lambda}=1 .
$$

If the Hermitian metric is not flat, the situation becomes harder. In the setup of closed manifolds M Braverman and T Kappeler performed a deformation procedure in [6, Section 11] and proved in this way the relation between the Ray-Singer norm and the refined analytic torsion in [6, Theorem 11.3].

Unfortunately the deformation argument is not local and the arguments in [6] do not apply in the setup of manifolds with boundary. Nevertheless we can derive appropriate result by relating our discussion to the closed double manifold.

Assume the metric structures $\left(g^{M}, h^{E}\right)$ to be product near the boundary $\partial M$. The issues related to the product structures are discussed in detail in [4, Section 2]. More precisely, we identify using the inward geodesic flow a collar neighborhood $U \subset M$ 
of the boundary $\partial M$ diffeomorphically with $[0, \epsilon) \times \partial M, \epsilon>0$. Explicitly we have the diffeomorphism

$$
\begin{aligned}
\phi^{-1}:[0, \epsilon) \times \partial M & \rightarrow U, \\
(t, p) & \mapsto \gamma_{p}(t),
\end{aligned}
$$

where $\gamma_{p}$ is the geodesic flow starting at $p \in \partial M$ and $\gamma_{p}(t)$ is the geodesics from $p$ of length $t \in[0, \epsilon)$. The metric $g^{M}$ is product near the boundary, if over $U$ it is given under the diffeomorphism $\phi: U \rightarrow[0, \epsilon) \times \partial M$ by

$$
\left.\phi_{*} g^{M}\right|_{U}=\left.d x^{2} \oplus g^{M}\right|_{\partial M} .
$$

The diffeomorphism $U \cong[0, \epsilon) \times \partial M$ shall be covered by a bundle isomorphism $\tilde{\phi}:\left.E\right|_{U} \rightarrow[0, \epsilon) \times\left. E\right|_{\partial M}$. The fiber metric $h^{E}$ is product near the boundary, if it is preserved by the bundle isomorphism, ie

$$
\left.\tilde{\phi}_{*} h^{E}\right|_{\{x\} \times \partial M}=\left.h^{E}\right|_{\partial M} .
$$

The assumption of product structures guarantees that the closed double manifold

$$
\mathbb{M}=M \cup_{\partial M} M
$$

is a smooth closed Riemannian manifold and the Hermitian vector bundle $\left(E, h^{E}\right)$ extends to a smooth Hermitian vector bundle $\left(\mathbb{E}, h^{\mathbb{E}}\right)$ over the manifold $\mathbb{M}$.

Moreover we assume the flat connection $\nabla$ on $E$ to be in temporal gauge. The precise definition of a connection in temporal gauge and the proof of the fact that each flat connection is gauge-equivalent to a flat connection in temporal gauge, are provided in [32].

The assumption on $\nabla$ to be a flat connection in temporal gauge is required in the present context to guarantee that $\nabla$ extends to a smooth flat connection $\mathbb{D}$ on $\mathbb{E}$, with

$$
\left.\mathbb{D}\right|_{M}=\nabla \text {. }
$$

Theorem 6.3 Let $\left(M^{m}, g^{M}\right)$ be an odd-dimensional oriented and compact smooth Riemannian manifold with boundary $\partial M$. Let $\left(E, \nabla, h^{E}\right)$ be a flat Hermitian vector bundle with the Hermitian metric $h^{E}$, not necessarily flat.

Assume the metric structures $\left(g^{M}, h^{E}\right)$ to be product and the flat connection $\nabla$ to be in temporal gauge near the boundary $\partial M$. Then

$$
\left\|\rho_{\mathrm{an}}(\nabla)\right\|_{\operatorname{det} H^{*}(\tilde{\mathcal{D}}, \tilde{\nabla})}^{\mathrm{RS}}=\exp \left(\pi \operatorname{Im} \eta\left(\mathcal{B}_{\mathrm{even}}\left(g^{M}\right)\right)\right) .
$$


Proof By assumption we obtain a closed Riemannian double manifold $\left(\mathbb{M}, g^{\mathbb{M}}\right)$ and a flat Hermitian vector bundle $\left(\mathbb{E}, \mathbb{D}, h^{\mathbb{E}}\right)$ over $\mathbb{M}$ with a flat Hermitian metric $h^{\mathbb{E}}$. Denote by $(\mathcal{D}, \mathbb{D})$ the unique boundary conditions (see Brüning and Lesch [9]) of the twisted de Rham complex $\left(\Omega^{*}(\mathbb{M}, \mathbb{E}), \mathbb{D}\right)$. Denote the closure of $\Omega^{*}(\mathbb{M}, \mathbb{E})$ with respect to the $L^{2}-$ scalar product defined by $g^{\mathbb{M}}$ and $h^{\mathbb{E}}$, by $L_{*}^{2}(\mathbb{M}, \mathbb{E})$.

The Riemannian metric $g^{\mathbb{M}}$ gives rise to the Hodge star operator $*$ and we set

$$
\mathbb{G}:=i^{r}(-1)^{k(k+1) / 2} *: \Omega^{k}(\mathbb{M}, \mathbb{E}) \rightarrow \Omega^{k-1}(\mathbb{M}, \mathbb{E}), \quad r:=(\operatorname{dim} M+1) / 2
$$

which extends to a self-adjoint involution on $L_{*}^{2}(\mathbb{M}, \mathbb{E})$. We define the odd signature operator $\mathbb{B}$ of the Hilbert complex $(\mathcal{D}, \mathbb{D})$ :

$$
\mathbb{B}:=\mathbb{G} \mathbb{D}+\mathbb{D} \mathbb{G} .
$$

This is precisely the odd-signature operator associated to the closed manifold $\mathbb{M}$, as used in the construction of $[8 ; 6]$.

Note that we now have two triples: the triple $(\mathbb{D}, \mathbb{G}, \mathbb{B})$ associated to the closed manifold $\mathbb{M}$ and the triple $(\widetilde{\nabla}, \widetilde{\Gamma}, \mathcal{B})$ associated to $(M, \partial M)$, as defined in Section 3. Consider now the diffeomorphic involution on the closed double

$$
\alpha: \mathbb{M} \rightarrow \mathbb{M},
$$

interchanging the two copies of $M$. It gives rise to an isomorphism of Hilbert complexes

$$
\alpha^{*}:(\mathcal{D}, \mathbb{D}) \rightarrow(\mathcal{D}, \mathbb{D}),
$$

which is an involution as well. We get a decomposition of $(\mathcal{D}, \mathbb{D})$ into the $( \pm 1)-$ eigenspaces of $\alpha^{*}$, which form subcomplexes of the total complex

$$
(\mathcal{D}, \mathbb{D})=\left(\mathcal{D}^{+}, \mathbb{D}^{+}\right) \oplus\left(\mathcal{D}^{-}, \mathbb{D}^{-}\right),
$$

where the upper indices \pm refer to the $( \pm 1)$-eigenspaces of $\alpha^{*}$, respectively.

The central property of the decomposition, by similar arguments as in [9, Theorem 4.1], lies in the observation

$$
\left.\mathcal{D}^{+}\right|_{M}=\mathcal{D}_{\max },\left.\quad \mathcal{D}^{-}\right|_{M}=\mathcal{D}_{\text {min }}
$$

By the symmetry of the elements in $\mathcal{D}^{ \pm}$we obtain the following natural isomorphism of complexes:

$$
\begin{gathered}
\Phi:(\mathcal{D}, \mathbb{D})=\left(\mathcal{D}^{+}, \mathbb{D}^{+}\right) \oplus\left(\mathcal{D}^{-}, \mathbb{D}^{-}\right) \rightarrow\left(\mathcal{D}_{\max }, \nabla_{\max }\right) \oplus\left(\mathcal{D}_{\min }, \nabla_{\min }\right), \\
\omega=\left.\left.\omega^{+} \oplus \omega^{-} \mapsto 2 \omega^{+}\right|_{M} \oplus 2 \omega^{-}\right|_{M},
\end{gathered}
$$


which extends to an isometry with respect to the natural $L^{2}$-structures. Using the relations

$$
\Phi \circ \mathbb{D} \circ \Phi^{-1}=\tilde{\nabla}, \quad \Phi \circ \mathbb{G} \circ \Phi^{-1}=\widetilde{\Gamma},
$$

we obtain with $\Delta$ and $\widetilde{\triangle}$, denoting respectively the Laplacians of the complexes $(\mathcal{D}, \mathbb{D})$ and $(\tilde{\mathcal{D}}, \tilde{\nabla}) \equiv\left(\mathcal{D}_{\min }, \nabla_{\min }\right) \oplus\left(\mathcal{D}_{\max }, \nabla_{\max }\right)$ :

$$
\begin{aligned}
\Phi \mathcal{D}(\mathbb{B})=\mathcal{D}(\mathcal{B}), & \Phi \circ \mathbb{B} \circ \Phi^{-1}=\mathcal{B}, \\
\Phi \mathcal{D}(\Delta)=\mathcal{D}(\tilde{\triangle}), & \Phi \circ \Delta \circ \Phi^{-1}=\widetilde{\triangle} .
\end{aligned}
$$

Hence the odd-signature operators $\mathbb{B}, \mathcal{B}$ as well as the Laplacians $\Delta, \widetilde{\triangle}$ are spectrally equivalent. Consider the spectral projections $\Pi_{\mathbb{B}^{2},[0, \lambda]}$ and $\Pi_{\mathcal{B}^{2},[0, \lambda]}, \lambda \geq 0$ of $\mathbb{B}$ and $\mathcal{B}$ respectively, associated to eigenvalues of absolute value in $[0, \lambda]$. By the spectral equivalence $\mathbb{B}$ and $\mathcal{B}$ we find

$$
\Phi \circ \Pi_{\mathbb{B}^{2},[0, \lambda]}=\Pi_{\mathcal{B}^{2},[0, \lambda]} \circ \Phi .
$$

Hence the isomorphism $\Phi$ reduces to an isomorphism of finite-dimensional complexes

$$
\Phi_{\lambda}:\left(\mathcal{D}_{[0, \lambda]}, \mathbb{D}_{[0, \lambda]}\right) \stackrel{\sim}{\rightarrow}\left(\tilde{\mathcal{D}}_{[0, \lambda]}, \tilde{\nabla}_{[0, \lambda]}\right),
$$

where

$$
\begin{aligned}
& \mathcal{D}_{[0, \lambda]}:=\mathcal{D} \cap \text { Image } \Pi_{\mathbb{B}^{2},[0, \lambda]}, \\
& \widetilde{\mathcal{D}}_{[0, \lambda]}:=\tilde{\mathcal{D}} \cap \text { Image } \Pi_{\mathcal{B}^{2},[0, \lambda]} .
\end{aligned}
$$

Moreover $\Phi_{\lambda}$ induces an isometric identification of the corresponding determinant lines, which we denote again by $\Phi_{\lambda}$, by a minor abuse of notation

$$
\Phi_{\lambda}: \operatorname{det}\left(\mathcal{D}_{[0, \lambda]}, \mathbb{D}_{[0, \lambda]}\right) \stackrel{\sim}{\rightarrow} \operatorname{det}\left(\tilde{\mathcal{D}}_{[0, \lambda]}, \tilde{\nabla}_{[0, \lambda]}\right),
$$

where we use the notation for determinant lines of finite-dimensional complexes in [6, Section 1.1]. By Corollary 3.16 we have the canonical identifications of determinant lines

$$
\begin{aligned}
& \operatorname{det}\left(\mathcal{D}_{[0, \lambda]}, \mathbb{D}_{[0, \lambda]}\right) \cong \operatorname{det} H^{*}(\mathcal{D}, \mathbb{D}), \\
& \operatorname{det}\left(\widetilde{\mathcal{D}}_{[0, \lambda]}, \widetilde{\nabla}_{[0, \lambda]}\right) \cong \operatorname{det} H^{*}(\widetilde{\mathcal{D}}, \widetilde{\nabla}),
\end{aligned}
$$

The determinant lines on the left hand side of both identifications carry the natural $L^{2}-$ Hilbert structure. Denote the norms on $\operatorname{det} H^{*}(\mathcal{D}, \mathbb{D})$ and $\operatorname{det} H^{*}(\widetilde{\mathcal{D}}, \widetilde{\nabla})$ which turn both identifications into isometries, by $\|\cdot\|_{\lambda}$ and $\|\cdot\|_{\lambda}^{\sim}$, respectively. Then we can view $\Phi_{\lambda}$ as

$$
\Phi_{\lambda}: \operatorname{det} H^{*}(\mathcal{D}, \mathbb{D}) \stackrel{\sim}{\rightarrow} \operatorname{det} H^{*}(\widetilde{\mathcal{D}}, \widetilde{\nabla}),
$$

isometric with respect to the Hilbert structures induced by $\|\cdot\|_{\lambda}$ and $\|\cdot\|_{\lambda}$. 
Finally, consider the refined torsion elements (not the refined analytic torsion) of the determinant lines, as defined in [6, Section 1.1] (see also (3-8)):

$$
\begin{gathered}
\rho_{[0, \lambda]}^{\mathbb{G}} \in \operatorname{det}\left(\mathcal{D}_{[0, \lambda]}, \mathbb{D}_{[0, \lambda]}\right) \cong \operatorname{det} H^{*}(\mathcal{D}, \mathbb{D}), \\
\rho_{[0, \lambda]}^{\widetilde{\Gamma}} \in \operatorname{det}\left(\widetilde{\mathcal{D}}_{[0, \lambda]}, \widetilde{\nabla}_{[0, \lambda]}\right) \cong \operatorname{det} H^{*}(\widetilde{\mathcal{D}}, \widetilde{\nabla}) .
\end{gathered}
$$

We infer from (6-13) the following relation:

$$
\Phi_{\lambda}\left(\rho_{[0, \lambda]}^{\mathbb{G}}\right)=\rho_{[0, \lambda]}^{\widetilde{\Gamma}}, \text { hence }\left\|\rho_{[0, \lambda]}^{\mathbb{G}}\right\|_{\lambda}=\left\|\rho_{[0, \lambda]}^{\tilde{\Gamma}_{\lambda}}\right\|_{\lambda}^{\sim} .
$$

Together with spectral equivalence of $\Delta$ and $\widetilde{\triangle}$, as well as of $\mathbb{B}$ and $\mathcal{B}$, with similar statements for constructions on trivial line bundles $M \times \mathbb{C}$ and $\mathbb{M} \times \mathbb{C}$, we finally obtain

$$
\left\|\rho_{\mathrm{an}}(\mathbb{D})\right\|_{\operatorname{det} H^{*}(\mathcal{D}, \mathbb{D})}^{\mathrm{RS}}=\left\|\rho_{\mathrm{an}}(\nabla)\right\|_{\operatorname{det} H^{*}(\tilde{\mathcal{D}}, \tilde{\nabla})}^{\mathrm{RS}},
$$

where $\rho_{\text {an }}(\mathbb{D})$ denotes the refined analytic torsion as defined by M Braverman and T Kappeler in [6] and $\rho_{\text {an }}(\nabla)$ denotes the refined analytic torsion in the sense of the present discussion.

The statement now follows from [6, Theorem 11.3].

\section{Open problems}

Ideal boundary conditions As explained in the introduction, the approach of Braverman and Kappeler in $[8 ; 6]$ requires ideal boundary conditions for the twisted de Rham complex, which turn it into a Fredholm complex with Poincare duality and further provide elliptic boundary conditions for the associated odd-signature operator, viewed as a map between the even forms. In our construction we pursued a different strategy, however the question about existence of such boundary conditions remains.

This question was partly discussed in [9]. In view of [9, Lemma 4.3] it is not even clear whether ideal boundary conditions exist, satisfying Poincare duality and providing a Fredholm complex. For the approach of Braverman and Kappeler we need even more: the ideal boundary conditions need to provide elliptic boundary conditions for the odd-signature operator. We arrive at the natural open question, whether such boundary conditions exist.

Conical singularities Another possible direction for the discussion of refined analytic torsion is the setup of compact manifolds with conical singularities. At the conical singularity the question of appropriate boundary conditions is discussed by Cheeger [14], as well as by Brüning and Lesch [10]. 
It turns out that on odd-dimensional manifolds with conical singularities the topological obstruction is given by $H^{v}(N)$, where $N$ is the base of the cone and $v=\operatorname{dim} N / 2$. If

$$
H^{v}(N)=0
$$

then all ideal boundary conditions coincide and the construction of Braverman and Kappeler [8; 6] goes through. Otherwise, see [14, page 580] for the choice of ideal boundary conditions satisfying Poincare duality.

Combinatorial counterpart Let us recall that the definition of the refined analytic torsion in $[8 ; 6]$ was partly motivated by providing analytic counterpart of the refined combinatorial torsion, introduced by V Turaev in [30].

In his work V Turaev introduced the notion of Euler structures and showed how it is applied to refine the concept of Reidemeister torsion by removing the ambiguities in choosing bases needed for construction. Moreover, Turaev observed in [31] that on three-manifolds a choice of an Euler structure is equivalent to a choice of a $\operatorname{Spin}^{c}-$ structure.

Both the Turaev torsion and the Braverman-Kappeler refined torsion are holomorphic functions on the space of representations of the fundamental group on $G L(n, \mathbb{C})$, which is a finite-dimensional algebraic variety. Using methods of complex analysis, Braverman and Kappeler computed the quotient between their and Turaev's construction.

A natural question is whether this procedure has an appropriate equivalent for our proposed refined analytic torsion on manifolds with boundary. In our view this question can be answered affirmatively.

Indeed, by similar arguments as in $[8 ; 6]$ the proposed refined analytic torsion on manifolds with boundary can also be viewed as an analytic function on the finitedimensional variety of representations of the fundamental group.

For the combinatorial counterpart note that $M$ Farber introduced in [15] the concept of Poincare-Reidemeister metric, where using Poincare duality in the similar spirit as in our construction, he constructed an invariantly defined Reidemeister torsion norm for nonunimodular representations. Further M Farber and V Turaev elaborated jointly in [16] the relation between their concepts and introduced the refinement of the Poincare-Reidemeister scalar product.

The construction in [15] extends naturally to manifolds with boundary by similar means as in our definition of refined analytic torsion. This provides a combinatorial torsion norm on compact manifolds, well-defined without unimodularity assumption. It can then be refined in the spirit of [16]. This would naturally provide the combinatorial counterpart for the presented refined analytic torsion. 
Complex-valued Ray-Singer torsion by Burghelea-Haller In the context of providing an analytic counterpart to the combinatorial refinement by V Turaev, the construction by D Burghelea and S Haller in [12] is of great interest. There an alternative definition of refined analytic torsion is considered and a conjecture on its relation to the Turaev torsion is formulated. This conjecture has been proved independently by Burghelea and Haller in [13] and Su and Zhang in [29].

The relationship of the construction by Burghelea and Haller with the refinement by Braverman and Kappeler is discussed in [7] and [5]. A natural question in the context of the present paper, whether any extension of the Burghelea-Haller torsion to manifolds with boundary is possible.

Gluing formula for refined analytic torsion A refinement of analytic Ray-Singer torsion should naturally resemble the striking property of the torsion invariant - its gluing property, established by S Vishik in [33] and W Lück in [23]. This result is surprising in view of the nonlocality of spectral invariants and is useful for computational issues.

In the subsequent paper [32] we solve the problem affirmatively in case of a Hermitian connection, presenting an explicit gluing formula for our construction. We use an isomorphism between determinant lines, which takes the inner symmetry of refined torsion elements into account and is not related to the isomorphism of Vishik [33]. Further we derive a gluing formula for the scalar analytic torsion.

\section{References}

[1] S Agmon, On the eigenfunctions and on the eigenvalues of general elliptic boundary value problems, Comm. Pure Appl. Math. 15 (1962) 119-147 MR0147774

[2] M F Atiyah, V K Patodi, I M Singer, Spectral asymmetry and Riemannian geometry. I, Math. Proc. Cambridge Philos. Soc. 77 (1975) 43-69 MR0397797

[3] N Berline, E Getzler, M Vergne, Heat kernels and Dirac operators, Grund. der Math. Wissenschaften 298, Springer, Berlin (1992) MR1215720

[4] B Booß-Bavnbek, M Lesch, C Zhu, The Calderon projection: new definition and applications arXiv:0803.4160

[5] M Braverman, T Kappeler, Comparison of the refined analytic and the BurgheleaHaller torsions, Ann. Inst. Fourier (Grenoble) 57 (2007) 2361-2387 MR2394545 Festival Yves Colin de Verdière

[6] M Braverman, $\mathbf{T}$ Kappeler, Refined analytic torsion as an element of the determinant line, Geom. Topol. 11 (2007) 139-213 MR2302591 
[7] M Braverman, T Kappeler, A canonical quadratic form on the determinant line of a flat vector bundle, Int. Math. Res. Not. (2008) Art. ID rnn030, 21pp MR2428856

[8] M Braverman, T Kappeler, Refined analytic torsion, J. Differential Geom. 78 (2008) 193-267 MR2394022

[9] J Brüning, M Lesch, Hilbert complexes, J. Funct. Anal. 108 (1992) 88-132 MR1174159

[10] J Brüning, M Lesch, Kähler-Hodge theory for conformal complex cones, Geom. Funct. Anal. 3 (1993) 439-473 MR1233862

[11] D Burghelea, L Friedlander, T Kappeler, Meyer-Vietoris type formula for determinants of elliptic differential operators, J. Funct. Anal. 107 (1992) 34-65 MR1165865

[12] D Burghelea, S Haller, Complex-valued Ray-Singer torsion, J. Funct. Anal. 248 (2007) 27-78 MR2329682

[13] D Burghelea, S Haller, Complex-valued Ray-Singer torsion II arXiv: math/0610875

[14] J Cheeger, Spectral geometry of singular Riemannian spaces, J. Differential Geom. 18 (1983) 575-657 (1984) MR730920

[15] M Farber, Combinatorial invariants computing the Ray-Singer analytic torsion, Differential Geom. Appl. 6 (1996) 351-366 MR1422341

[16] M Farber, V Turaev, Poincaré-Reidemeister metric, Euler structures, and torsion, J. Reine Angew. Math. 520 (2000) 195-225 MR1748274

[17] P B Gilkey, Invariance theory, the heat equation, and the Atiyah-Singer index theorem, second edition, Studies in Advanced Math., CRC Press, Boca Raton, FL (1995) MR1396308

[18] P B Gilkey, L Smith, The eta invariant for a class of elliptic boundary value problems, Comm. Pure Appl. Math. 36 (1983) 85-131 MR680084

[19] PB Gilkey, L Smith, The twisted index problem for manifolds with boundary, J. Differential Geom. 18 (1983) 393-444 MR723812

[20] R-T Huang, Refined analytic torsion: comparison theorems and examples, Illinois J. Math. 51 (2007) 1309-1327 MR2417429

[21] T Kato, Perturbation theory for linear operators, second edition, Grund. der Math. Wissenschaften 132, Springer, Berlin (1976) MR0407617

[22] F F Knudsen, D Mumford, The projectivity of the moduli space of stable curves. I. Preliminaries on "det” and "Div”, Math. Scand. 39 (1976) 19-55 MR0437541

[23] W Lück, Analytic and topological torsion for manifolds with boundary and symmetry, J. Differential Geom. 37 (1993) 263-322 MR1205447

[24] W Müller, Analytic torsion and R-torsion for unimodular representations, J. Amer. Math. Soc. 6 (1993) 721-753 MR1189689 
[25] L Paquet, Problèmes mixtes pour le système de Maxwell, Ann. Fac. Sci. Toulouse Math. (5) 4 (1982) 103-141 MR687546

[26] D B Ray, I M Singer, $R$-torsion and the Laplacian on Riemannian manifolds, Advances in Math. 7 (1971) 145-210 MR0295381

[27] R Seeley, Analytic extension of the trace associated with elliptic boundary problems, Amer. J. Math. 91 (1969) 963-983 MR0265968

[28] R Seeley, The resolvent of an elliptic boundary problem, Amer. J. Math. 91 (1969) 889-920 MR0265764

[29] G Su, W Zhang, A Cheeger-Müller theorem for symmetric bilinear torsions, Chin. Ann. Math. Ser. B 29 (2008) 385-424 MR2429629

[30] V Turaev, Euler structures, nonsingular vector fields, and Reidemeister-type torsions, Izv. Akad. Nauk SSSR Ser. Mat. 53 (1989) 607-643, 672 MR1013714

[31] V Turaev, Torsion invariants of $\operatorname{Spin}^{c}$-structures on 3-manifolds, Math. Res. Lett. 4 (1997) 679-695 MR1484699

[32] B Vertman, Gluing formula for refined analytic torsion arXiv:0808.0451

[33] S M Vishik, Generalized Ray-Singer conjecture. I. A manifold with a smooth boundary, Comm. Math. Phys. 167 (1995) 1-102 MR1316501

Department of Mathematics, University of Bonn

Beringstr. 6, 53115 Bonn, Germany

vertman@math.uni-bonn.de

Proposed: Tobias Colding

Seconded: David Gabai, Gang Tian
Received: 17 August 2008

Revised: 17 March 2009 